\title{
An inexact derivative-free Levenberg-Marquardt method for linear inequality constrained nonlinear systems under local error bound conditions
}

\author{
Peng Wang ${ }^{\mathrm{a}}$, Detong $\mathrm{Zhu}{ }^{\mathrm{b}, *}$ \\ ${ }^{a}$ Mathematics and Statistics College, Hainan Normal University, Hainan 570203, China \\ ${ }^{b}$ Mathematics and Science College, Shanghai Normal University, Shanghai 200234, China
}

\begin{abstract}
In this paper, a derivative-free affine scaling inexact Levenberg-Marquardt method with interior backtracking line search technique is considered for solving linear inequality constrainted nonlinear systems. The proposed algorithm is designed to take advantage of the problem structure by building polynomial interpolation models for each function of nonlinear systems subject to the linear inequality constraints on variables. Each iterate switches to backtracking step generated by affine scaling inexact Levenberg-Marquardt method and satisfies strict interior point feasibility by line search backtracking technique. Under local error bounded assumption, the method is superlinear and quadratic convergent on $F(x)$. The results of numerical experiments are reported to show the effectiveness of the proposed algorithm.
\end{abstract}

Keywords: derivative-free optimization, Levenberg-Marquardt method, inexact, system of nonlinear equations, interior point, affine scaling

2010 MSC: 49M37, 65K05, 90C30, 90C56

\section{Introduction}

In this paper, we consider a derivative-free optimization algorithm for the solution of nonlinear systems subject to linear inequality on variables. The general nonlinear systems is of the following form:

$$
\text { (P) } \quad F(x)=0, \quad x \in \Omega=\{x \mid A x \geq b\},
$$

where $F(x)=\left(f_{1}(x), f_{2}(x), \cdots, f_{q}(x)\right)^{T}$, and $f_{i}: \mathbf{R}^{n} \rightarrow \mathbf{R}, i=1,2, \cdots, q$, are general nonlinear twice continuously differentiable functions, but none of their first-order or second-order derivatives is explicitly available. $\Omega=\{x \mid A x \geq$ $b\}$ is the feasible region where $A=\left[a_{1}^{T}, a_{2}^{T}, \cdots, a_{l}^{T}\right]^{T} \in \mathbf{R}^{l \times n}$. Moreover, it is assumed that the interior of the feasible region $\operatorname{int}(\Omega)=\{x \mid A x>b\}$ is not empty.

The problem have many engineering applications. One of such applications is in power engineering. The operation of a power system is described by a system of nonlinear equations called the power flow equations (see $[9,27])$. The power flow equations are constrained by some operating constraints. Moreover, $(P)$ provides a unifying framework for the study of complementarity problems of different kinds [26] and it is also important in engineering and economic applications by finding the solutions of certain equations [13].

In the last decade, Levenberg-Marquardt methods $[18,21]$ have been useful tools for solving $(P)$. For results on the local convergence we refer to $[10,11,14,17,31]$ and several globalization techniques have been suggested in $[1,12,20]$. Zhu [32] suggested the affine scaling interior Levenberg-Marquardt method for solving bound-constrainted semismooth equations. In the Levenberg-Marquardt method [28], it is assumed that the method is solved exactly at every iteration. However, it is expensive to take the exact solution of method for large-scale problems, and hence

\footnotetext{
The authors gratefully acknowledge the partial supports of the National Science Foundation Grant (11371253) of China.

* Corresponding author.

Email addresses: pengwang621@163.com (Peng Wang ), dtzhu@shnu.edu.cn (Detong Zhu )
} 
it is often effective to use inexact methods that find an approximate solution satisfying some appropriate conditions. For example, Dan, Yamashita and Fukushima [6] introduced the inexact Levenberg-Marquardt method. The inexact Levenberg-Marquardt method did not require computing exact search directions, and the method had a superlinear rate of convergence under the local error bound assumption. Behling and Fischer [2] suggested an inexact constrained Levenberg-Marquardt method. They showed that the best results known for the unconstrained case also hold for the constrained Levenberg-Marquardt method. At the same time, the influence of the regularization parameter on the level of inexactness and the convergence rate was described.

In many practices, it is often impossible or impractical to obtain the derivative of $F(x)$, so in some cases, derivativefree algorithms are used. There are some papers introducing the derivative-free algorithm. Glad and Goldst [15] had suggested minimizing regression quadratic models as in directional direct-search methods. Elster and Neumaier [8] developed an algorithm based on the minimization of quadratic regression models within trust regions. Conn, Scheinberg, and Toint [5] introduced the interpolation-based derivative-free trust-region method and showed that the algorithm converged to first-order critical points. Marazzi and Nocedal [19] also introduced the wedge algorithm in 2002. The method generated a model that interpolates the objective function at a set of sample points, and the trust regions was used to promote convergence. The step-generation subproblem ensured that all the iterates satisfied a geometric condition. The sample points were updated using a scheme that improved the accuracy of the interpolation model when needed. Two versions of the method were presented in the paper, one used linear models and the other used quadratic models. Wang and Zhu [25] introduced an affine scaling derivative-free trust-region method for solving nonlinear systems subject to linear inequality constraints.

The proposed algorithm is designed to take advantage of the problem structure by building polynomial interpolation models for each function in the nonlinear system function $F$. By using both trust-region strategy and interior line search backtracking technique, each iteration switches to backtracking step generated by the trust region subproblem and satisfies strict interior point feasibility by line search backtracking technique. Under reasonable conditions, the global convergence and fast local convergence rate of the proposed algorithm are established. Unlike other modelbased methods in the literature, our algorithm takes full advantages of the structure of problem by building polynomial interpolation models for each function $f_{i}(x)$ in the problem. Moreover, applying Powell's minimum Frobenius norm updating technique [23], the algorithm uses $2 n+1$ sampling points which is less than $\frac{(n+1)(n+2)}{2}$ required for building a fully quadratic model to asymptotically build at least fully linear models for each of the nonlinear functions in the problem. Levenberg-Marquardt algorithms have to solve complicated quadratic programming subproblem at each iteration. In the case where $\Omega$ is polyhedral, if iterative point $x_{k} \in$ int $\Omega$, it is difficult to compute the search direction $d_{k}$ such that $x_{k}+d_{k} \in$ int $\Omega$. Hence, the total computational effort for completing one iteration might be expensive and difficult. In order to overcome this shortcoming, line search technique is often used in [33]. In this paper, we would like to study the local convergence behavior of the algorithm and establish local superlinear and quadratic convergence of the algorithm under some local error bound conditions which are considerably weaker than a non-singularity assumption on the Jacobian.

This paper is organized as follow. In section 2, we introduce some basic properties of the interpolation models associated with the algorithm. The derivative-free affine scaling inexact Levenberg-Marquardt algorithm with backtracking interior point technique for solving problem $(P)$ is given in section 3 . In the section 4 , we introduce some model properties and show the global convergence of the algorithm. We establish the local superlinear convergence of the algorithm for zero residual problems in the section 5. Finally, some numerical results are given in section 6.

Notation: In this paper, the norm $\|\cdot\|$ is the 2-norm for a vector and the induced 2-norm for a matrix. $|S|$ denotes the cardinality of set $S$, and $B(z, \Delta)$ denoted the closed ball which the center at $z$, with radius $\Delta>0 . P_{n}^{d}$ denoted the space of all polynomials of degree $n \leq d$ in $\mathbf{R}^{n}$. Some constants used in this paper are defined here for convenience.

$\kappa_{e f}, \hat{\kappa}_{e f}: \quad$ error in the function value;

$\kappa_{e g}, \hat{\kappa}_{e g}: \quad$ error in the gradient;

$\kappa_{m}: \quad$ bound on all the separate models;

$\kappa_{g}: \quad$ bound on the gradients of all the separate models;

$\kappa_{H}^{\phi}: \quad$ bound on the Hessian of $\phi$;

$\kappa_{D}$ : $\quad$ bound on the inverse of affine-scaling matrix $D(x)$. 


\section{Model properties}

In this section, we first consider the following problem:

$$
\begin{aligned}
& \min \Phi(x)=\frac{1}{2} \sum_{i=1}^{q} f_{i}^{2}(x)=\frac{1}{2}\|F(x)\|^{2} \\
& \text { s.t. } x \in \Omega=\{x \mid A x \geq b\}
\end{aligned}
$$

where the norm $\|\cdot\|$ is the standard Euclidian norm. It is clear that problem $(P)$ is equivalent to problem $(S)$ in the zero residual case.

In fact, every solution $x^{*}$ of the problem $(P)$ is a global minimum of the problem $(S)$. Conversely, if $x^{*}$ is a minimum of the problem $(S)$ such that $\Phi\left(x^{*}\right)=0$, then $x^{*}$ is also a solution of the problem $(P)$.

For the convergence of algorithm, we define a $\Lambda$-pointed set $B \in \mathbf{R}^{n}$ from [7]. The definition is as follows:

Definition 2.1 Let $\Lambda>0$ and $P$ be a space of polynomials on $\mathbf{R}^{n}$ with a basis $\varphi=\left\{\varphi_{0}(x), \varphi_{1}(x), \cdots, \varphi_{p}(x)\right\}$. Then a set $Y=\left\{y^{0}, y^{1}, \cdots, y^{p}\right\}$ is said to be $\Lambda$-poised in $B$ for $P$ (in the interpolation sense) if and only if, for any $x \in B \subset \mathbf{R}^{n}$, there is $\lambda(x) \in \mathbf{R}^{p+1}$ such that

$$
\sum_{i=0}^{p} \lambda_{i}(x) \varphi\left(y^{i}\right)=\varphi(x) \text { with }\|\lambda(x)\|_{\infty} \leq \Lambda .
$$

Other equivalent definitions of a $\Lambda$-poised set are given in [23] that the above definition does not depend on the choice of the basis $\varphi(x)$ as long as it spans the same space $P$. Since we are mostly interested in using models which are defined on the interpolation sets whose cardinality is smaller than that required for fully determined quadratic interpolation, we will also use the definition of a $\Lambda$-poised set $Y \subseteq \mathbf{R}^{n}$ for minimum Frobenius norm interpolation.

Definition 2.2 Let $\Lambda>0$ and a set $B \in \mathbf{R}^{n}$ be given. Let $\hat{\varphi}=\left\{\hat{\varphi}_{0}(x), \hat{\varphi}_{1}(x), \cdots, \hat{\varphi}_{C_{n+2}^{2}-1}(x)\right\}$ be the natural basis of monomials of $P_{n}^{2}$ (order by degree). A poised set $Y=\left\{y^{0}, y^{1}, \cdots, y^{p}\right\}$, with $C_{n+1}^{1} \leq p+1 \leq C_{n+2}^{2}$, is said to be $\Lambda$-poised in $B$ (in the minimum Frobenius norm sense ) if and only if, for any $x \in B$, there is a solution $\lambda(x) \in \mathbf{R}^{p+1}$ of

$$
\begin{aligned}
& \min \sum_{j=C_{n+1}^{1}}^{C_{n+2}^{2}-1}\left(\sum_{i=0}^{p} \lambda_{i}(x) \hat{\varphi}_{j}\left(y_{i}\right)-\hat{\varphi}_{j}(x)\right)^{2} \\
& \text { s.t. } \quad \sum_{i=0}^{p} \lambda_{i}(x) \hat{\varphi}_{j}\left(y_{i}\right)=\hat{\varphi}_{j}(x), \quad 0 \leq j \leq n
\end{aligned}
$$

such that $\|\lambda(x)\|_{\infty} \leq \Lambda$.

Suppose we have a $\Lambda$-poised set $Y \subseteq B(z, \Delta)$. For each $i=1,2, \cdots, q$, let $m_{i} \in P_{n}^{2}$ be a polynomial interpolating model of $f_{i}(x)$ on $Y$. Substituting $f_{i}(x)$ by $m_{i}(x)$ in the objective function of problem $(S)$, we obtain that

$$
M(x)=\frac{1}{2}\|m(x)\|^{2}=\frac{1}{2} \sum_{i=1}^{q} m_{i}^{2}(x) .
$$

Now taking the second-order Taylor expansion of $M(x)$ at a point $y$ in terms of variables, we obtain the local quadratic model $\phi(y, s)$ of $\Phi(\cdot)$ around $y$ as

$$
\phi(y, s)=c_{\phi}(y)+g_{\phi}(y)^{T} s+\frac{1}{2} s^{T} H_{\phi}(y) s
$$

where $m(y)=\left(m_{1}(y), m_{2}(y), \cdots, m_{q}(y)\right)^{T}, J(y)=\nabla m(y)=\left(\nabla m_{1}(y), \nabla m_{2}(y), \cdots, \nabla m_{q}(y)\right)^{T}, c_{\phi}(y)=\frac{1}{2} m(y)^{T} m(y)$, $g_{\phi}(y)=J(y)^{T} m(y)$ and $H_{\phi}(y)=J(y)^{T} J(y)$. Here $m_{i}(\cdot) \in P_{n}^{2}, i=1,2, \cdots, q$, are the polynomial interpolating models of $f_{i}(\cdot)$ on Y.

Assume the resulting iterates $x_{k} \in \operatorname{int}(\Omega)$ and $y_{k} \in Y_{k} \subseteq B\left(x_{k}, \Delta_{k}\right)$, we define

$$
g_{\phi_{k}}=g_{\phi}\left(x_{k}\right) \text {. }
$$


In order to give the trust-region subproblem, scaling matrix of the problem will be introduced. Let $x_{k}$ be the current strictly feasible iterate and $\lambda_{k}$ be an approximation to the Lagrangian multiplier vector of $(S)$, the scaling matrix $D_{k}$ and the diagonal matrix $C_{k}$ are defined as follows:

$$
D_{k}=\operatorname{diag}\left\{A x_{k}-b\right\} \text {, and } C_{k}=\operatorname{diag}\left\{\left|\lambda_{k}\right|\right\} .
$$

Optimality conditions for problem $(S)$ are well established. A feasible point $x^{*} \in \Omega$ is said to be stationary point for problem $(S)$ which is called the first order necessary condition, if there exists a Lagrangian multiplier vector $0 \leq \lambda^{*} \in \mathbf{R}^{l}$ such that

$$
\operatorname{diag}\left\{A x^{*}-b\right\} \lambda^{*}=0 \text {, and } \nabla \Phi\left(x^{*}\right)-A^{T} \lambda^{*}=0 .
$$

Strict complementarity is said to hold at $x^{*}$ if at least one of the two inequalities $a_{i}^{T} x^{*}-b_{i}>0$ and $\lambda_{i}^{*}>0 \quad(i=$ $1,2, \cdots, l)$ holds, where $b_{i}$ and $\lambda_{i}^{*}$ are the $i$ th component of the vectors $b$ and $\lambda^{*}$, respectively. Ignoring primal and dual feasibility of the inequality constraints, the first order necessary condition of problem $(S)$ can be expressed by the following system of nonlinear equations,

$$
\begin{aligned}
\nabla \Phi(x)-A^{T} \lambda & =0, \\
\operatorname{diag}\{A x-b\} \lambda & =0 .
\end{aligned}
$$

The augmented affine scaling trust-region subproblem at the $k$ th iteration is defined

$$
\begin{aligned}
& \min \phi_{k}(d)=\phi\left(x_{k}, d\right)+\frac{1}{2} d^{T}\left(A^{T} D_{k}^{-1} C_{k} A\right) d \\
& \text { s.t. }\left\|\left(d ; D_{k}^{-\frac{1}{2}} A d\right)\right\| \leq \Delta_{k},
\end{aligned}
$$

where $\Delta_{k}$ is the trust-region radius.

The Levenberg-Marquardt method is a modified trust region strategy that is designed to maintain advantages of trust region method. Considering the Kuhn-Tucker conditions of $\left(S_{k}\right)$, we build the global minimum of the affine scaling quadratic model

$$
\begin{aligned}
\min \hat{\phi}_{k}(d) & =\frac{1}{2}\left\|m\left(x_{k}\right)+\nabla m\left(x_{k}\right) d\right\|^{2}+\frac{1}{2} \hat{d}^{T} C_{k} \hat{d}+\frac{1}{2} \mu_{k}\left\|\left(d^{T}, \hat{d}^{T}\right)\right\|^{2}+\lambda_{k}^{T}\left(A d-D^{\frac{1}{2}} \hat{d}\right) \\
& =\frac{1}{2}\left\|m\left(x_{k}\right)+\nabla m\left(x_{k}\right) d\right\|^{2}+\frac{1}{2} d^{T}\left(A^{T} D_{k}^{-1} C_{k} A\right) d+\frac{1}{2} \mu_{k}\left(d^{T} d+d^{T} A^{T} D_{k}^{-1} A d\right),
\end{aligned}
$$

where $\hat{d}_{k}=D_{k}^{-\frac{1}{2}} A d, \lambda_{k}$ is Lagrange multiplier and $\mu_{k}$ is a positive parameter such that $\hat{\phi}_{k}(d)$ is a strict convex function. Let $\left(d_{k}, \hat{d}_{k}\right)$ denote a solution to $\left(\hat{S}_{k}\right)$ and define

$$
\hat{M}_{k}=\left[\begin{array}{cc}
H_{\phi}\left(x_{k}\right) & 0 \\
0 & C_{k}
\end{array}\right]
$$

The first order necessary conditions of $\left(\hat{S}_{k}\right)$ imply that

$$
\left(\hat{M}_{k}+\mu_{k} I\right)\left(\begin{array}{c}
d_{k} \\
\hat{d}_{k}
\end{array}\right)=-\left(\begin{array}{c}
g_{\phi_{k}} \\
0
\end{array}\right)+\left[\begin{array}{c}
A^{T} \\
-D_{k}^{\frac{1}{2}}
\end{array}\right] \hat{\lambda}_{k},
$$

where the multipliers $\hat{\lambda}_{k}$ satisfy the normal equation $\left[A A^{T}+D_{k}\right] \hat{\lambda}_{k}=-A g_{\phi_{k}}$.

Since $\nabla \Phi\left(x_{k}\right)$ is not explicitly available, we use $g_{\phi_{k}}$ instead of $\nabla \Phi\left(x_{k}\right)$ in (2.2). Let $\hat{\Gamma}_{k}=-\left(g_{\phi_{k}}-A^{T} \hat{\lambda}_{k}\right)$, then

$$
g_{\phi_{k}}^{T} \hat{\Gamma}_{k}=-\left(\left\|g_{\phi_{k}}-A^{T} \hat{\lambda}_{k}\right\|^{2}+\left\|D_{k}^{\frac{1}{2}} \hat{\lambda}_{k}\right\|^{2}\right) .
$$

Equation (2.5) suggests that a "good" decrease of the quadratic objective function in $\left(S_{k}\right)$ along the gradient $g_{\phi_{k}}$ can lead to dual feasibility:

$$
\lim _{k \rightarrow \infty}\left\|g_{\phi_{k}}-A^{T} \hat{\lambda}_{k}\right\|=0, \text { and } \lim _{k \rightarrow \infty}\left\|D_{k}^{\frac{1}{2}} \hat{\lambda}_{k}\right\|=0
$$


Set

$$
M_{k}=\nabla m\left(x_{k}\right)^{T} \nabla m\left(x_{k}\right)+A^{T} D_{k}^{-1} C_{k} A,
$$

since $\hat{\phi}_{k}(d)$ is a strictly convex function, $d$ is also the global minimum of the subproblem $\left(\hat{S}_{k}\right)$ which is in fact equivalent to solving the following affine scaling Levenberg-Marquardt-type equation

$$
\left[M_{k}+\mu_{k}\left(I+A^{T} D_{k}^{-1} A\right)\right] d=-g_{\phi_{k}},
$$

where $g_{\phi_{k}}=\nabla m\left(x_{k}\right)^{T} m\left(x_{k}\right)$.

For large-scale problem, solving subproblem $\left(\hat{S}_{k}\right)$ exactly is expensive. Hence, it is often effective to use inexact method that find an approximate solution satisfying some appropriate conditions. Therefore, we consider the inexact method that uses an approximate solution $d_{k}$ of subproblem $\left(\hat{S}_{k}\right)$. Let $d_{k}$ be the approximate solution of subproblem $\left(\hat{S}_{k}\right)$, then the vector $r_{k}$ is defined by

$$
r_{k}=\left[M_{k}+\mu_{k}\left(I+A^{T} D_{k}^{-1} A\right)\right] d_{k}+g_{\phi_{k}},
$$

where the vector $r_{k}$ is a residual vector associated with $d_{k}$.

Moreover, in order to maintain the strict interior feasibility, we give a backtracking step along $d_{k}$ which is the approximate solution of the quadratic affine scaling subproblem $\left(\hat{S}_{k}\right)$.

\section{The algorithm}

In this section, we describe an inexact derivative-free Levenberg-Marquardt algorithm with strict interior feasible backtracking line search technique for solving linearly constrained problem $(P)$. In this algorithm, the number of points in the sampling set is an integer constant $N_{P} \in\left[C_{n+1}^{1}, C_{n+2}^{2}\right]$, and we denote the resulting iterates by $x_{k}$.

\section{Algorithm}

\section{Initialization Step}

Choose the starting strict feasibility interior point $x_{0} \in \operatorname{int}(\Omega) \subseteq \mathbf{R}^{n}, 0<\rho_{0} \leq \bar{\Delta}_{0} \leq \Delta_{0} \leq \Delta_{\max }$, and the number of sampling points $N_{P} \geq C_{n+1}^{1}$. Let $Y_{0}$ be the initial interpolation points set, and $x_{0} \in Y_{0} \subset B\left(x_{0}, \bar{\Delta}_{0}\right), \theta_{0}=0.02, \gamma \in(0,1)$, $\omega \in(0,1)$ and $\varepsilon_{\beta} \in(0,1)$, set $k=0$, go to the main step.

\section{Main Step}

1. Calculate

$$
\begin{aligned}
g_{\phi_{k}} & =\nabla m\left(x_{k}\right)^{T} m\left(x_{k}\right), \\
\hat{\Gamma}_{k} & =-\left(g_{\phi_{k}}-A^{T} \hat{\lambda}_{k}\right),
\end{aligned}
$$

where $x_{k} \in Y_{k}$ and $\hat{\lambda}_{k}$ satisfies the normal equation $\left[A A^{T}+D_{k}\right] \hat{\lambda}_{k}=-A g_{\phi_{k}}$.

2. If $\left|g_{\phi_{k}}^{T} \hat{\Gamma}_{k}\right|^{\frac{1}{2}} \leq \varepsilon_{\beta}$ and $Y_{k}$ is $\Lambda$-poised in $B\left(x_{k}, \bar{\Delta}_{k}\right)$ if needed, where $\bar{\Delta}_{k}=\min \left\{\Delta_{k},\left|g_{\phi_{k}}^{T} \hat{\Gamma}_{k}\right|^{\frac{1}{2}}\right\}$, then stop.

Else modifying $Y_{k}$ to make sure $Y_{k}$ is $\Lambda$-poised in $B\left(x_{k}, \bar{\Delta}_{k}\right)$ and $x_{k} \in Y_{k}$, determine $m\left(x_{k}\right)$ if $Y_{k}$ has changed and return to 1 .

3. Find an approximate solution $d_{k}$ of the system of linear equations

$$
\left[M_{k}+\mu_{k}\left(I+A^{T} D_{k}^{-1} A\right)\right] d=-g_{\phi_{k}}+r_{k}, \quad \text { with } \quad\left\|r_{k}\right\| \leq \eta_{k}\left\|\nabla m\left(x_{k}\right)^{T} m\left(x_{k}\right)\right\| .
$$

4. Choose $\alpha_{k}=1, \omega, \omega^{2}, \cdots$, until the following inequality is satisfied:

$$
\begin{aligned}
& \Phi\left(x_{k}+\alpha_{k} d_{k}\right) \leq\left(1-\alpha_{k} \gamma\right) \Phi\left(x_{k}\right), \\
& x_{k}+\alpha_{k} d_{k} \in \Omega .
\end{aligned}
$$


5. Let

$$
h_{k}= \begin{cases}\alpha_{k} d_{k}, & \text { if } x_{k}+\alpha_{k} d_{k} \in \operatorname{int}(\Omega), \\ \theta_{k} \alpha_{k} d_{k}, & \text { otherwise }\end{cases}
$$

where $\theta_{k} \in\left(\theta_{0}, 1\right]$ and $\theta_{k}-1=O\left(\left\|d_{k}\right\|\right)$.

6. Compute $\Phi\left(x_{k}+h_{k}\right)$ and $\Phi\left(x_{k}+d_{k}\right)$.

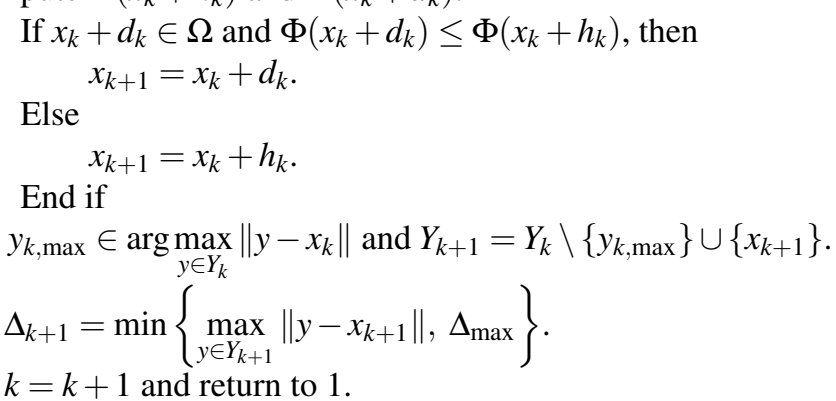

Set $k=k+1$ and return to 1

Remark 1 The scalar $\alpha_{k}$ is given in Step 4, denotes the step size along the direction $d_{k}$ to the boundary of linear inequality constraints,

$$
\alpha_{k} \leq \min \left\{-\frac{a_{i}^{T} x_{k}-b_{i}}{a_{i}^{T} d_{k}} \mid-\frac{a_{i}^{T} x_{k}-b_{i}}{a_{i}^{T} d_{k}}>0, i=1,2, \cdots, l\right\},
$$

with $\alpha_{k}=+\infty$ if $-\left(a_{i}^{T} x_{k}-b_{i}\right) /\left(a_{i}^{T} d_{k}\right) \leq 0$ for all $i$. A key property of the scalar $\alpha_{k}$ is that an arbitrary step $\alpha_{k} d_{k}$ to the point $x_{k}+\alpha_{k} d_{k}$ does not violate any linear inequality constraints. To see this, first observe that if $-\left(a_{i}^{T} x_{k}-\right.$ $\left.b_{i}\right) /\left(a_{i}^{T} d_{k}\right) \leq 0$ for some $i=1,2, \cdots, m$, and hence $a_{i}^{T} x_{k}-b_{i}>0$ implies $a_{i}^{T} d_{k}>0$. Therefore, for all $\alpha_{k} \in(0,+\infty)$,

$$
a_{i}^{T}\left(x_{k}+\alpha_{k} d_{k}\right)-b_{i}=a_{i}^{T} x_{k}-b_{i}+\alpha_{k} a_{i}^{T} d_{k}>0,
$$

which means the $i$ th linear strick inequality constraint holds.

If $\alpha_{k}=\left(\left(a_{i}^{T} x_{k}-b_{i}\right) /\left(a_{i}^{T} d_{k}\right)\right)>0$ for some $i$, and hence $\left(a_{i}^{T} x_{k}-b_{i}\right)>0$ implies $a_{i}^{T} d_{k}<0$. We have that from $\alpha_{k} \leq-\left(a_{i}^{T} x_{k}-b_{i}\right) /\left(a_{i}^{T} d_{k}\right)$,

$$
a_{i}^{T} x_{k}-b_{i} \leq-\alpha_{k} a_{i}^{T} d_{k} .
$$

Hence, (22) and (23) mean that no matter what cases the inequality $a_{i}^{T}\left(x_{k}+\alpha_{k} d_{k}\right) \geq b_{i}$ for all any $i=1,2, \cdots, l$ holds. Remark 2 There are some suitable forcing terms $\eta_{k}$ in Step 3 of the algorithm. In this paper, we can choose

$$
\eta_{k} \leq \mu_{k}\left\|m\left(x_{k}\right)\right\|, \quad k=1,2, \cdots .
$$

\section{Model properties and global convergence}

In this section, we present a few lemmas which provide some basic properties of the interpolation models used in the algorithm, and these properties will be used on global convergence. Firstly, we show, under our assumptions of $\Lambda$-poisedness of the sample set $Y$, an interpolating polynomial is at least local fully linear on $Y$.

Lemma 4.1[4] Given any $\Delta>0, z \in \mathbf{R}^{n}$, and $Y=\left\{y_{0}, y_{1}, y_{2}, \cdots, y_{p}\right\} \subset B(z, \Delta) \Lambda$-poised in $B(z, \Delta)$ with $C_{n+1}^{1} \leq|Y| \leq$ $C_{n+2}^{2}$, let $m(\cdot) \in P_{n}^{2}$ be a interpolating polynomial of $f$ on $Y$, i.e.,

$$
m\left(y_{i}\right)=f\left(y_{i}\right), i=1,2, \cdots,|Y| .
$$

If $f_{i}: \mathbf{R}^{n} \rightarrow \mathbf{R}, i=1, \cdots, q$ is continuously differentiable and $\nabla f_{i}, i=1, \cdots, q$ is Lipschitz continuous with Lipschtiz constant $L$ in a open set containing $B(z, \Delta)$, then, for any $s \in B(0, \Delta)$ and $y \in Y_{k}$, we have that

$$
\begin{aligned}
\left\|\nabla f_{i}(z+s)-\nabla m_{i}(z+s)\right\| & \leq \hat{\kappa}_{e g}(n, \Lambda)\left(\left\|\nabla^{2} m(y)\right\|+L\right) \Delta, \\
\left|f_{i}(z+s)-m_{i}(z+s)\right| & \leq \hat{\kappa}_{e f}(n, \Lambda)\left(\left\|\nabla^{2} m(y)\right\|+L\right) \Delta^{2},
\end{aligned}
$$


where $\hat{k}_{e g}$ and $\hat{k}_{e f}$ are positive constants depending only on $n$ and $\Lambda$.

Based on the above lemma, there exist positive constants $\kappa_{e g}$ and $\kappa_{e f}$ which depending only on $n, f_{i}, F$, and $\max \left\{\left\|\nabla^{2} m_{i}\right\|, i=1,2, \cdots, q\right\}$ such that, for any $s \in B(0, \Delta)$

$$
\begin{aligned}
\left\|\nabla f_{i}(z+s)-\nabla m_{i}(z+s)\right\| & \leq \kappa_{e g} \Delta, \\
\left|f_{i}(z+s)-m_{i}(z+s)\right| & \leq \kappa_{e f} \Delta^{2}
\end{aligned}
$$

for all $i=1,2, \cdots, q$ hold, where $m_{i}(x)$ is the polynomial interpolating model of $f_{i}(x)$ on $Y$. Furthermore, if $\zeta$ is a fixed constant for all iterations and $\max \left\{\left\|\nabla^{2} m_{i}(y)\right\|, i=1,2, \cdots, q\right\} \leq \zeta$, then $\kappa_{e g}$ and $\kappa_{e f}$ will be fixed constants, too.

Now, we define $\operatorname{conv}\left(L_{e n l}\left(x_{0}\right)\right)$ to be the convex hull of $L_{e n l}\left(x_{0}\right)$ with

$$
L_{e n l}\left(x_{0}\right)=\bigcup_{x \in L\left(x_{0}\right)} B\left(x, \Delta_{\max }\right) \text { and } L\left(x_{0}\right)=\left\{x \in R^{n}: \Phi(x) \leq \Phi\left(x_{0}\right)\right\},
$$

where $\Phi(x)$ is defined in problem $(S)$. The following assumptions are needed for the convergence of algorithm in this paper.

Assumption 4.1 Assume that $F$ is twice continuously differentiable and the level set $A\left(x_{0}\right)=\left\{x \in \mathbf{R}^{n}: \Phi(x) \leq\right.$ $\left.\Phi\left(x_{0}\right), A x \geq b\right\}$ is bounded.

Assumption 4.2 There exists a positive constant $\kappa_{H}$, independent of the iteration number $k$ in the algorithm, such that if $m_{i}, i=1,2, \cdots, q$, is the polynomial interpolating model of $f_{i}$ on a $\Lambda$-poised sampling set $Y_{k}$ constructed as in the algorithm, then, for any $y \in Y_{k}$,

$$
\left\|\nabla^{2} m_{i}(y)\right\| \leq \kappa_{H}
$$

for all $i=1,2, \cdots, q$.

Assumption 4.3 There exists a constant $\kappa_{D}>0$ such that $\left\|D_{k}\right\| \leq \kappa_{D}$ for all $x \in A\left(x_{0}\right)$.

Assumption 4.4 $\left[A-D(x)^{\frac{1}{2}}\right]$ is assumed to have full row rank for all $x \in A\left(x_{0}\right)$.

Through first-order or second-order derivatives of $F$ are not explicitly available, according to assumption 4.1, the following lemma holds.

Lemma 4.2[29] Under Assumption 4.1, there exist positive constants $L_{0}, L_{1}, L_{2}, L_{3}$ and $L_{4}$ such that

$$
\begin{aligned}
\|F(x)\| & \leq L_{0}, \\
\|\nabla F(x)\| & \leq L_{1}, \\
\|F(x)-F(y)\| & \leq L_{2}\|x-y\|, \\
\|\nabla F(x)-\nabla F(y)\| & \leq L_{3}\|x-y\|, \\
\left\|\nabla^{2} f_{i}(x)\right\| & \leq L_{4}, i=1,2, \cdots, q
\end{aligned}
$$

for any $x, y \in \operatorname{conv}\left(L_{\text {enl }}\left(x_{0}\right)\right)$.

With Assumptions 4.1 and 4.2, we show that the models used in the algorithm would satisfy the following bounds. Lemma 4.3[29] Under Assumptions 4.1 and 4.2, there exist positive constants $k_{m}$ and $k_{g}$, independent of $k$, such that if $m_{i}, i=1,2, \cdots, q$, is the polynomial interpolating model of $f_{i}$ on a $\Lambda$-poised sampling set $Y_{k}$ constructed as in the algorithm, then, for any $y \in Y_{k}$,

$$
\left|m_{i}(y)\right| \leq \kappa_{m} \text { and }\left\|\nabla m_{i}(y)\right\| \leq \kappa_{g}
$$

for all $i=1,2, \cdots, q$. Hence, there exists a constant $\kappa_{H}^{\phi}$ such that

$$
\left\|H_{\phi}(y)\right\| \leq \kappa_{H}^{\phi}
$$

for any $y \in Y_{k}$.

Next, we establish the global convergence of the algorithm. Firstly, we will show the definition of the upper Dini directional derivative of $\Phi(x)$. According to Lemma 4.2, we know that $F(x)$ is Lipshitz continuous, so is $\Phi(x)$. Hence upper Dini directional derivative of $\Phi(x)$ exists at every $x \in \Omega$ and along every direction $d \in \mathbf{R}^{n}$

$$
\Phi^{D}(x ; d)=\limsup _{t \downarrow 0} \frac{\Phi(x+t d)-\Phi(x)}{t} .
$$


Now, we show that the algorithm will produce an iterate $x_{k+1}=x_{k}+\alpha_{k} d_{k}$ in a finite number of backtracking steps in Step 4 of algorithm.

Lemma 4.4 Under the Assumptions 4.1-4.4, if

$$
\Phi^{D}\left(x_{k} ; d_{k}\right) \leq-\gamma F\left(x_{k}\right)^{T} F\left(x_{k}\right)
$$

for all large enough $k$, then the algorithm will produce an iterate $x_{k+1}=x_{k}+\alpha_{k} d_{k}$ in a finite number of backtracking steps in Step 4 of the algorithm.

Proof Let $d_{k}$ be an arbitrary solution of (3.1). We show that there exists a number $\bar{\alpha}>0$ such that for all $\alpha_{k} \in(0, \bar{\alpha}]$,

$$
\Phi\left(x_{k}+\alpha_{k} d_{k}\right)-\Phi\left(x_{k}\right) \leq-\alpha_{k} \gamma \Phi\left(x_{k}\right) .
$$

Assume the contrary. Then, there exists a sequence $\left\{\alpha_{i}\right\}$ of positive numbers converging to 0 , such that for each $i$,

$$
\Phi\left(x_{k}+\alpha_{i} d_{k}\right)-\Phi\left(x_{k}\right)>-\alpha_{i} \gamma \Phi\left(x_{k}\right) \text {. }
$$

Dividing both sides by $\alpha_{i}$, passing to the upper limit $i \rightarrow \infty$ and using the definition of the Dini derivative (4.3) of $\Phi(x)$, we obtain that

$$
\Phi^{D}\left(x_{k} ; d_{k}\right) \geq-\gamma \Phi\left(x_{k}\right)
$$

On the other hand, according to (4.4), we have that

$$
\Phi^{D}\left(x_{k} ; d_{k}\right) \leq-\gamma F\left(x_{k}\right)^{T} F\left(x_{k}\right)=-2 \gamma \Phi\left(x_{k}\right),
$$

which is a contradiction. Hence, the algorithm will produce an iterate $x_{k+1}=x_{k}+\alpha_{k} d_{k}$ in a finite number of backtracking steps in Step 4 of the algorithm.

Remark 3 The assumption $\Phi^{D}\left(x_{k} ; d_{k}\right) \leq-\gamma F\left(x_{k}\right)^{T} F\left(x_{k}\right)$ ensures that every solution $d_{k}$ of (3.1) will yield a descent direction for the norm function $\Phi$ at $x_{k}$. Similar assumption was used by Martínez and Qi [22] to prove global convergence of some inexact Newton method. Following Martínez and Qi we note that " $\gamma$-assumption" is the minimal assumption which is sufficient and qualitatively weaker assumption cannot support the globalization. For instance, according to the definition of $\Phi^{D}\left(x_{k}, d_{k}\right)$ and $\Phi^{D}\left(x_{k} ; d_{k}\right) \leq-\gamma F\left(x_{k}\right)^{T} F\left(x_{k}\right)$, we have that

$$
\frac{\Phi\left(x_{k}+t d_{k}\right)-\Phi\left(x_{k}\right)}{t} \leq-\gamma F\left(x_{k}\right)^{T} F\left(x_{k}\right)
$$

which imply

$$
t \nabla \Phi\left(x_{k}\right)^{T} d_{k}+o(t) \leq-\gamma F\left(x_{k}\right)^{T} F\left(x_{k}\right)
$$

By (4.5), we have that

$$
t\left(\nabla \Phi\left(x_{k}\right)^{T} d_{k}+\gamma F\left(x_{k}\right)^{T} F\left(x_{k}\right)\right)+o(t) \leq 0 .
$$

Hence, if $\nabla \Phi\left(x_{k}\right) d_{k}+F\left(x_{k}\right)^{T} F\left(x_{k}\right)=0$, then we obtain (4.4).

Let $\Gamma_{k_{i}}=-\left(\nabla \Phi\left(x_{k_{i}}\right)-A^{T} \lambda_{k_{i}}\right)$ and $\hat{\Gamma}_{k_{i}}=-\left(g_{\phi_{k_{i}}}-A^{T} \hat{\lambda}_{k_{i}}\right)$, where $\lambda_{k_{i}}$ and $\hat{\lambda}_{k_{i}}$ are the Lagrange multiplier corresponding to $\nabla \Phi\left(x_{k_{i}}\right)$ and $g_{\phi_{k_{i}}}$, respectively. Similar to the proof Lemma 4.6 in [25], the following lemma shows the relationship between $\left|g_{\phi_{k_{i}}}^{T} \hat{\Gamma}_{k_{i}}\right|^{\frac{1}{2}}$ and $\left|\nabla \Phi\left(x_{k_{i}}\right)^{T} \Gamma_{k_{i}}\right|$.

Lemma 4.5 Under the Assumptions 4.1-4.4, for any subsequence $\left\{x_{k_{i}}\right\}$ generated by the algorithm, if $\lim _{i \rightarrow \infty}\left|g_{\phi_{k_{i}}}^{T} \hat{\Gamma}_{k_{i}}\right|^{\frac{1}{2}}=$ 0 , then $\lim _{i \rightarrow \infty}\left|\nabla \Phi\left(x_{k_{i}}\right)^{T} \Gamma_{k_{i}}\right|^{\frac{1}{2}}=0$.

Theorem 4.6 Under the Assumptions 4.1-4.4, let $\left\{x_{k}\right\}$ be a sequence generated by the proposed algorithm. If the conditions of Lemma 4.4 hold, then

$$
\liminf _{k \rightarrow \infty}\left|g_{\phi_{k}}^{T} \hat{\Gamma}_{k}\right|^{\frac{1}{2}}=0
$$


Proof Assume the conclusion of the theorem is not true, then there exists some $\varepsilon>\varepsilon_{\beta}>0$ such that

$$
\left|g_{\phi_{k}}^{T} \hat{\Gamma}_{k}\right| \geq \varepsilon, k=1,2, \cdots .
$$

By Assumption 4.4, we have that $A A^{T}+D_{k}$ is positive definite matrix, i.e., $\lambda_{\min }\left[A A^{T}+D_{k}\right]>0$, where $\lambda_{\min }\left[A A^{T}+\right.$ $\left.D_{k}\right]$ is the minimum eigenvalue of matrix $A A^{T}+D_{k}$ ). By (4.7), we know that the algorithm can not stop. According to Lemma 4.4, we have that $\left\{\Phi\left(x_{k}\right)\right\}$ is monotonically decreasing, i.e., there exists an index $K_{0}$ such that

$$
\Phi\left(x_{k}\right)=\frac{1}{2}\left\|F\left(x_{k}\right)\right\|^{2}<\frac{\varepsilon^{2}}{2 q^{2} \kappa_{g}^{2}\left(\frac{\kappa_{D}^{\frac{1}{2}}\|A\|+\|A\|^{2}}{\lambda_{\min }\left[A A^{T}+D_{k}\right]+1}\right)^{2}}
$$

when $k>K_{0}$, which means $\left\|F\left(x_{k}\right)\right\|<\varepsilon / q \kappa_{g}\left(\frac{\kappa_{D}^{\frac{1}{2}}\|A\|+\|A\|^{2}}{\lambda_{\min }\left[A A^{T}+D_{k}\right]+1}\right)$ for all $k>K_{0}$.

According to the definition of $\left|g_{\phi_{k}}^{T} \hat{\Gamma}_{k}\right|$ and $\left[A A^{T}+D_{k}\right] \hat{\lambda}_{k}=-g_{\phi_{k}}$, we have that

$$
\begin{aligned}
\left|g_{\phi_{k}}^{T} \hat{\Gamma}_{k}\right|^{\frac{1}{2}} & =\sqrt{\left\|g_{\phi_{k}}-A \hat{\lambda}_{k}\right\|^{2}+\left\|D_{k}^{\frac{1}{2}} \hat{\lambda}_{k}\right\|^{2}} \\
& \leq\left\|g_{\phi_{k}}-A \hat{\lambda}_{k}\right\|+\left\|D_{k}^{\frac{1}{2}} \hat{\lambda}_{k}\right\| \\
& \leq\left\|D_{k}^{\frac{1}{2}}\left(A A^{T}+D_{k}\right)^{-1} g_{\phi_{k}}\right\|+\left\|g_{\phi_{k}}\right\|+\left\|A\left(A A^{T}+D_{k}\right)^{-1} A g_{\phi_{k}}\right\| \\
& \leq\left(\frac{\left\|D_{k}\right\|^{\frac{1}{2}}\|A\|}{\lambda_{\min }\left[A A^{T}+D_{k}\right]}+\frac{\|A\|^{2}}{\lambda_{\min }\left[A A^{T}+D_{k}\right]}+1\right)\left\|\nabla m\left(x_{k}\right)\right\|\left\|F\left(x_{k}\right)\right\| \\
& \leq q \kappa_{g}\left(\frac{\kappa_{D}^{\frac{1}{2}}\|A\|+\|A\|^{2}}{\lambda_{\min }\left[A A^{T}+D_{k}\right]+1}\right)\left\|F\left(x_{k}\right)\right\| .
\end{aligned}
$$

From (4.7), (4.8) and (4.9), we have that

$$
\varepsilon \leq\left|g_{\phi_{k}}^{T} \hat{\Gamma}_{k}\right|^{\frac{1}{2}} \leq q \kappa_{g}\left(\frac{\kappa_{D}^{\frac{1}{2}}\|A\|+\|A\|^{2}}{\lambda_{\min }\left[A A^{T}+D_{k}\right]+1}\right)\left\|F\left(x_{k}\right)\right\|<\varepsilon,
$$

which is a contradiction and hence the conclusion of the theorem is true.

\section{Local convergence}

In this section, we give the local convergence of the algorithm. Under some assumptions, we can take superlinear convergence of the algorithm. Firstly, some important assumptions are given.

Assumption 5.1 Assume that problem $(P)$ is a zero residual problem, i.e.,

$$
F\left(x^{*}\right)=0, \text { for all } x^{*} \in X^{*} \subseteq \Omega,
$$

where $X^{*} \in \mathbf{R}^{n}$ is the solution set of $(P)$.

We can obviously see that $X^{*}$ is closed by the continuity of $F(x)$. So, $X^{*}$ is nonempty that for any $x \in \mathbf{R}^{n}$, and there exists a $\bar{x} \in X^{*}$ such that

$$
\operatorname{dist}\left(x, X^{*}\right)=\min _{y \in X^{*}}\|x-y\|=\|\bar{x}-x\| .
$$

According to Lemma 4.5 and Theorem 4.6, we know that $\nabla \Phi\left(x_{k}\right)^{T} \Gamma_{k}=-\nabla \Phi\left(x_{k}\right)^{T}\left(\nabla \Phi\left(x_{k}\right)^{T}-A \lambda_{k}\right) \rightarrow 0$ as $k \rightarrow \infty$. By the definition of $\nabla \Phi\left(x_{k}\right)$, we have that $F\left(x_{k}\right)^{T}\left(\nabla F\left(x_{k}\right) \nabla F\left(x_{k}\right)^{T} F\left(x_{k}\right)-\nabla F\left(x_{k}\right) A \lambda_{k}\right) \rightarrow 0$. Because $\lambda_{k}=$ 
$-\left[A A^{T}+D_{k}\right]^{-1} A \nabla F\left(x_{k}\right)^{T} F\left(x_{k}\right)$, we have that $F\left(x_{k}\right)^{T}\left(\nabla F\left(x_{k}\right) \nabla F\left(x_{k}\right)^{T}+\nabla F\left(x_{k}\right) A\left[A A^{T}+D_{k}\right]^{-1} A \nabla F\left(x_{k}\right)^{T}\right) F\left(x_{k}\right) \rightarrow$ 0. If $\nabla F\left(x_{k}\right) \nabla F\left(x_{k}\right)^{T}+\nabla F\left(x_{k}\right) A\left[A A^{T}+D_{k}\right]^{-1} A \nabla F\left(x_{k}\right)^{T}$ is a nonsingular matrix, then $F\left(x_{k}\right) \rightarrow 0$ as $k \rightarrow \infty$. Similar to Assumption 3.3 in [30], to discuss the local convergence properties of the algorithm, we assume that the sequence $\left\{x_{k}\right\}$ generated by the algorithm converges to the solution set $X^{*}$. Thus, we have the following assumption.

Assumption 5.2 Assume that the sequence of $\left\{x_{k}\right\}$ generated by the algorithm converges to the solution set $X^{*}$, i.e.,

$$
\lim _{k \rightarrow \infty} \operatorname{dist}\left(x_{k}, X^{*}\right)=0 .
$$

According to the local error bound assumption of Levenberg-Marquardt algorithm in [14], we assume that after the linear transformation of $\bar{x}_{k}-x_{k},\left\|D_{k}^{-\frac{1}{2}} A\left(\bar{x}_{k}-x_{k}\right)\right\|$ is controlled by the $\theta\left\|F\left(x_{k}\right)\right\|$. Specially, When the problem $(P)$ satisfies nondegenerate hypothesis in [33], i.e., if $\left|g_{\phi}\left(x^{*}\right)^{T} \hat{\Gamma}\left(x^{*}\right)\right|^{\frac{1}{2}}=0$, then $A x^{*}-b>0$ hold and we can show $D_{k}^{-\frac{1}{2}}$ is bounded for large enough $k$, the assumption will be not needed.

Assumption 5.3 Assume that

$$
\left\|D_{k}^{-\frac{1}{2}} A\left(\bar{x}_{k}-x_{k}\right)\right\| \leq \theta\left\|F\left(x_{k}\right)\right\|
$$

for all sufficiently large $k$, where $\theta \in(0,1)$.

The following assumption is concerned with the choice of the parameter $\mu_{k}$ used in the algorithm.

Assumption 5.4 Assume that the parameter $\mu_{k}$ is chosen to satisfy

$$
\mu_{k}=\zeta\left|g_{\phi_{k}}^{T} \hat{\Gamma}_{k}\right|^{\frac{\delta}{2}},
$$

where $\zeta$ is a positive constant and $\delta \in[1,2]$.

The Assumption 5.4 shows that the choice of the parameter $\mu_{k}$ is related to $\left|g_{\phi_{k}}^{T} \hat{\Gamma}_{k}\right|$. It is changed with the changing of $\left|g_{\phi_{k}}^{T} \hat{\Gamma}_{k}\right|$ in the algorithm. For the local convergence of the algorithm, we choose the parameter $\mu_{k}$ which is $\frac{\delta}{2}$ times of $\left|g_{\phi_{k}}^{T} \hat{\Gamma}_{k}\right|$. The parameter $\mu_{k}$ will tend to 0 if $\left|g_{\phi_{k}}^{T} \hat{\Gamma}_{k}\right|$ tends to 0 . By (2.8), we can obtain that residual vector $r_{k}$ is also controlled by $\mu_{k}$. The residual vector $r_{k} \rightarrow M_{k} d_{k}+g_{\phi_{k}}$ as $\mu_{k} \rightarrow 0$.

Suppose $x^{*}$ is a limit point of sequence $\left\{x_{k}\right\}$, by Assumption 5.1 and (4.9), we know that $\left|g_{\phi}\left(x^{*}\right)^{T} \hat{\Gamma}\left(x^{*}\right)\right|^{\frac{1}{2}}=0$, where $\hat{\Gamma}\left(x^{*}\right)=-\left(g_{\phi}\left(x^{*}\right)-A^{T} \hat{\lambda}\left(x^{*}\right)\right)$ and the multipliers $\hat{\lambda}\left(x^{*}\right)$ satisfies the normal equation $\left[A A^{T}+D\left(x^{*}\right)\right] \hat{\lambda}\left(x^{*}\right)=$ $-A g_{\phi}\left(x^{*}\right)$. According to Lemma 4.5 , we have that $\left|\nabla \Phi\left(x^{*}\right)^{T} \Gamma\left(x^{*}\right)\right|^{\frac{1}{2}}=0$ where $\Gamma\left(x^{*}\right)=-\left(\nabla \Phi\left(x^{*}\right)-A^{T} \lambda\left(x^{*}\right)\right)$ and the multipliers $\lambda\left(x^{*}\right)$ satisfies the normal equation $\left[A A^{T}+D\left(x^{*}\right)\right] \lambda\left(x^{*}\right)=-A \nabla \Phi\left(x^{*}\right)$. For the local convergence of the algorithm, we need the following local error bound assumption.

Assumption 5.5 Assume that $\left|\nabla \Phi(x)^{T} \Gamma(x)\right|^{\frac{1}{2}}$ provides a local error bound on $X^{*}$, i.e., there exists a positive constant $\rho>0$ such that

$$
\left|\nabla \Phi(x)^{T} \Gamma(x)\right|^{\frac{1}{2}} \geq \rho \operatorname{dist}\left(x, X^{*}\right),
$$

where $\operatorname{dist}\left(x, X^{*}\right)=\inf _{y \in X^{*}}\|x-y\|$ and $\Gamma(x)=-\left(\nabla \Phi(x)-A^{T} \lambda(x)\right), \lambda(x)$ is a Lagrange multiplier associated with conditions (2.2)-(2.3).

In Assumption 5.5, we give the local error bound conditions (5.4) instead of the local error bound conditions $\|F(x)\| \geq \rho \operatorname{dist}\left(x, X^{*}\right)$. Now, we show that the algorithm is superlinearly convergent.

Lemma 5.1 Under Assumptions 4.1-4.4, 5.1-5.2 and 5.4-5.5, we have that

$$
\lim _{k \rightarrow \infty}\left|\nabla \Phi\left(x_{k}\right)^{T} \Gamma_{k}\right|^{\frac{1}{2}}=0,
$$

where $\Gamma_{k}=-\left(\nabla \Phi\left(x_{k}\right)-A \lambda_{k}\right)$ and $\lambda_{k}$ is the Lagrange multiplier associated with conditions (2.2)-(2.3).

Proof By Lemma 4.2, we have that

$$
\begin{aligned}
\left|\nabla \Phi\left(x_{k}\right)^{T} \Gamma_{k}\right| & \leq\left\|\nabla \Phi\left(x_{k}\right)\right\|\left\|\Gamma_{k}\right\| \\
& \leq\left\|\nabla F\left(x_{k}\right)\right\|\left\|F\left(x_{k}\right)\right\|\left\|\Gamma_{k}\right\| \\
& \leq L_{1} L_{2}\left\|\bar{x}_{k}-x_{k}\right\|\left\|\Gamma_{k}\right\|,
\end{aligned}
$$


here and in what follows $\bar{x}_{k}$ is defined as in (5.1). According to the definition of $\Gamma_{k}$ and the Assumption 4.4, the Lagrangian multipliers $\lambda_{k}$ can be computed via $\left[A A^{T}+D_{k}\right] \lambda_{k}=A \nabla \Phi\left(x_{\phi_{k}}\right)$. Because $X^{*} \subseteq L\left(x_{0}\right)$, we have that $A A^{T}+$ $D\left(\bar{x}_{k}\right)$ is also a positive definite matrix, then there exists a positive constant $\kappa_{\Gamma}$ such that $\left\|\Gamma_{k}\right\| \leq \kappa_{\Gamma}$.

By (5.6), we have that

$$
\left|\nabla \Phi\left(x_{k}\right)^{T} \Gamma_{k}\right| \leq \kappa_{\Gamma} L_{1} L_{2}\left\|\bar{x}_{k}-x_{k}\right\|
$$

Hence, by Assumption 5.2, we have that $\lim _{k \rightarrow \infty}\left|\nabla \Phi\left(x_{k}\right)^{T} \Gamma_{k}\right|^{\frac{1}{2}}=0$.

The Assumptions 4.1 and 4.3 imply that there exists $\kappa_{C}>0$ such that $\left\|C_{k}\right\| \leq \kappa_{C}$. Next, for $d_{k}$ in Step 3 of the algorithm, we have the following lemma.

Lemma 5.2 Under Assumptions 4.1-4.4 and 5.1-5.5, for large enough $k$, there exists a positive constant $\hat{\kappa}$ such that

$$
\left\|d_{k}\right\| \leq \hat{\kappa} \operatorname{dist}\left(x_{k}, X^{*}\right)
$$

where $x_{k}$ was calculated by the algorithm.

Proof Let $s_{k}=\bar{x}_{k}-x_{k}$ and $\left\|\bar{x}_{k}-x_{k}\right\|$ is sufficiently small when $k$ is sufficiently large. Assume $\tilde{d}_{k}$ is the optimal solution of problem $\left(\hat{S}_{k}\right)$, because $A^{T} D_{k}^{-1} C_{k} A$ is a positive semi-definite matrix, we have that

$$
\begin{aligned}
\hat{\phi}_{k}\left(\tilde{d}_{k}\right) & =\frac{1}{2}\left\|m\left(x_{k}\right)+\nabla m\left(x_{k}\right) \tilde{d}_{k}\right\|^{2}+\frac{1}{2} \tilde{d}_{k}^{T} A^{T} D_{k}^{-1}\left(C_{k}+\mu_{k} I\right) A \tilde{d}_{k}+\frac{1}{2} \mu_{k}\left\|\tilde{d}_{k}\right\|^{2} \\
& \geq \frac{\mu_{k}}{2}\left\|\tilde{d}_{k}\right\|^{2} .
\end{aligned}
$$

Using the Taylor formula, we have that

$$
m\left(\bar{x}_{k}\right)=m\left(x_{k}\right)+\nabla m\left(x_{k}\right) s_{k}+\frac{1}{2} s_{k}^{T} \nabla^{2} m\left(\xi_{k}\right) s_{k},
$$

where $\xi_{k} \in\left(\bar{x}_{k}, x_{k}\right)$.

According to Assumption 4.2, by (5.9), we have used $0=f_{i}\left(\bar{x}_{k}\right)=m_{i}\left(\bar{x}_{k}\right)=m_{i}\left(x_{k}\right)+\nabla m\left(x_{k}\right)^{T} s_{k}+\frac{1}{2} s_{k}^{T} \nabla m_{i}\left(\xi_{k}\right) s_{k}$ and thus $\left\|m_{i}\left(x_{k}\right)+\nabla m_{i}\left(x_{k}\right)^{T} s_{k}\right\| \leq \frac{1}{2} \kappa_{H}\left\|s_{k}\right\|^{2}$, so we have that

$$
\left\|m\left(x_{k}\right)+\nabla m\left(x_{k}\right) s_{k}\right\| \leq \frac{\sqrt{q} \kappa_{H}}{2}\left\|s_{k}\right\|^{2}
$$

By Lemma 4.2 and Lemma 4.3, we have that

$$
\begin{aligned}
\left\|\nabla \Phi\left(x_{k}\right)-g_{\phi_{k}}\right\| & =\left\|\nabla F\left(x_{k}\right)^{T} F\left(x_{k}\right)-\nabla m\left(x_{k}\right)^{T} m\left(x_{k}\right)\right\| \\
& \leq\left\|F\left(x_{k}\right)\right\|\left\|\nabla F\left(x_{k}\right)-\nabla m\left(x_{k}\right)\right\| \\
& \leq\left\|F\left(x_{k}\right)\right\|\left(\left\|\nabla F\left(x_{k}\right)\right\|+\left\|\nabla m\left(x_{k}\right)\right\|\right) \\
& \leq q \kappa_{g} L_{1} L_{2}\left\|x_{k}-\bar{x}_{k}\right\| .
\end{aligned}
$$

According to Assumption 5.2 and (5.10), we know that $\left\|\nabla \Phi\left(x_{k}\right)-g_{\phi_{k}}\right\| \rightarrow 0$ for large enough $k$, by Assumption 4.4, we have that $A^{T} A+D_{k}$ is a positive definite matrix, because $\left[A^{T} A+D_{k}\right] \hat{\lambda}_{k}=g_{\phi_{k}}$ and $\left[A^{T} A+D_{k}\right] \lambda_{k}=\nabla \Phi\left(x_{k}\right)$, we obtain that $\left\|\hat{\lambda}_{k}-\lambda_{k}\right\| \rightarrow 0$.

By Assumption 4.4, $\left[A^{T} A+D_{k}\right] \hat{\lambda}_{k}=A g_{\phi_{k}}$ and the definition of matrix $C_{k}$, we have that there exists a positive constant $\theta_{1}$ such that $\left\|C_{k}\right\| \leq \theta_{1}\left\|g_{\phi_{k}}\right\|$. Because $\left\|g_{\phi_{k}}\right\|=\left\|\nabla m\left(x_{k}\right) m\left(x_{k}\right)\right\| \leq q \kappa_{g}\left\|m\left(x_{k}\right)\right\|$, we have that $\left\|C_{k}\right\| \leq$ $q \kappa_{g} \theta_{1}\left\|m\left(x_{k}\right)\right\|$. According to the definition of $\tilde{\phi}_{k}(x)$, Assumption 5.4 and Lemma 4.2, we have that

$$
\begin{aligned}
\tilde{\phi}_{k}\left(s_{k}\right) & =\frac{1}{2}\left\|m\left(x_{k}\right)+\nabla m\left(x_{k}\right) s_{k}\right\|^{2}+\frac{1}{2} s_{k}^{T} A^{T} D_{k}^{-1}\left(C_{k}+\mu_{k} I\right) A s_{k}+\frac{1}{2} \mu_{k}\left\|s_{k}\right\|^{2} \\
& \leq \frac{\left(\sqrt{q} \kappa_{H}\right)^{2}}{8}\left\|s_{k}\right\|^{4}+\frac{\theta^{2} L_{2}^{2}\left\|C_{k}\right\|}{2}\left\|s_{k}\right\|^{2}+\frac{1}{2} \mu_{k}\left(\theta^{2} L_{2}^{2}+1\right)\left\|s_{k}\right\|^{2} \\
& \leq \frac{\left(\sqrt{q} \kappa_{H}\right)^{2}}{8}\left\|s_{k}\right\|^{4}+\frac{q \kappa_{g} \theta^{2} L_{2}^{3} \theta_{1}}{2}\left\|s_{k}\right\|^{3}+\frac{1}{2} \mu_{k}\left(\theta^{2} L_{2}^{2}+1\right)\left\|s_{k}\right\|^{2}
\end{aligned}
$$


Because $\tilde{d}_{k}$ is the optimal solution of problem $\left(\hat{S}_{k}\right)$, we have that

$$
\tilde{\phi}_{k}\left(\tilde{d}_{k}\right) \leq \tilde{\phi}_{k}\left(s_{k}\right) .
$$

According to Assumption 5.3, combining (5.8), (5.11) and (5.12), we obtain that

$$
\frac{\zeta}{2}\left|g_{\phi_{k}}^{T} \hat{\Gamma}_{k}\right|^{\frac{\delta}{2}}\left\|\tilde{d}_{k}\right\|^{2} \leq \frac{\left(\sqrt{q} \kappa_{H}\right)^{2}}{8}\left\|s_{k}\right\|^{4}+\frac{q \kappa_{g} \theta^{2} L_{2}^{3} \theta_{1}}{2}\left\|s_{k}\right\|^{3}+2 \mu_{k}\left(\theta^{2} L_{2}^{2}+1\right)\left\|s_{k}\right\|^{2} .
$$

Similar to the proof Lemma 4.6 in [25], we have that $\left\|\nabla \Phi\left(x_{k}\right) \Gamma_{k}-g_{\phi_{k}}^{T} \hat{\Gamma}_{k}\right\| \rightarrow 0$ holds for large enough $k$, so we have that

$$
\begin{aligned}
\rho^{2} \operatorname{dist}\left(x_{k}, X^{*}\right)^{2} & =\rho^{2}\left\|s_{k}\right\|^{2} \\
& \leq\left|\nabla \Phi\left(x_{k}\right) \Gamma_{k}\right| \\
& \leq\left|\nabla \Phi\left(x_{k}\right) \Gamma_{k}-g_{\phi_{k}}^{T} \hat{\Gamma}_{k}\right|+\left|g_{\phi_{k}}^{T} \hat{\Gamma}_{k}\right| \\
& \leq 4\left|g_{\phi_{k}}^{T} \hat{\Gamma}_{k}\right| .
\end{aligned}
$$

By (5.14), we obtain that $\rho\left\|s_{k}\right\| \leq 2\left|g_{\phi_{k}}^{T} \hat{\Gamma}_{k}\right|^{\frac{1}{2}}$. From (5.13) and $\mu_{k}=\frac{\zeta}{2}\left|g_{\phi_{k}}^{T} \hat{\Gamma}_{k}\right|^{\frac{\delta}{2}}$, we have that

$$
\left\|\tilde{d}_{k}\right\|^{2} \leq \frac{\left(\sqrt{q} \kappa_{H}\right)^{2}}{4 \zeta(2 \rho)^{\delta}}\left\|s_{k}\right\|^{2}+\frac{q \kappa_{g} \theta^{2} L_{2}^{3} \theta_{1}}{\zeta(2 \rho)^{\delta}}\left\|s_{k}\right\|^{3-\frac{\delta}{2}}+4\left(\theta^{2} L_{2}^{2}+1\right)\left\|s_{k}\right\|^{2} .
$$

By (5.15), we have that

$$
\left\|\tilde{d}_{k}\right\| \leq \theta_{2}\left\|s_{k}\right\|,
$$

where $\theta_{2}=2 \max \left\{\sqrt{\left(4 \theta^{2} L_{2}^{2}+1\right)+\frac{\left(\sqrt{q} \kappa_{H}\right)^{2}}{4 \zeta(2 \rho)^{\delta}}}, \sqrt{\frac{q \kappa_{g} \theta^{2} L_{2}^{3} \theta_{1}}{\zeta(2 \rho)^{\delta}}}\right\}$.

By (2.7) and (2.8), we have that

$$
\tilde{d}_{k}=d_{k}-\left[M_{k}+\mu_{k}\left(I+A^{T} D_{k}^{-1} A\right)\right]^{-1} r_{k} .
$$

By (3.1), we know that

$$
\left\|r_{k}\right\| \leq\left\|\nabla m\left(x_{k}\right) m\left(x_{k}\right)\right\| \eta_{k} .
$$

According to the definition of $M_{k}, C_{k}$ and $D_{k}^{-\frac{1}{2}}$, we know that $d^{T} M_{k} d \geq 0$ and $d^{T} \mu_{k} A^{T} D_{k}^{-1} A d \geq 0$ for all nonzero $d \in \mathbf{R}^{n}$, then the matrix $M_{k}+\mu_{k} A^{T} D_{k}^{-1} A$ is a positive semidefinite matrix. Because the eigenvalues of $M_{k}+\mu_{k} A^{T} D_{k}^{-1} A$ are nonnegative, then $\left\|\left[M_{k}+\mu_{k}\left(I+A^{T} D_{k}^{-1} A\right)\right]^{-1}\right\|=\frac{1}{\lambda+\mu_{k}} \leq \mu_{k}^{-1}$, where $\tilde{\lambda}$ is the minimum eigenvalue of $M_{k}+$ $\mu_{k} A^{T} D_{k}^{-1} A$. From (3.7), (5.17) and (5.18), we have that

$$
\begin{aligned}
\left\|\tilde{d}_{k}\right\| & =\left\|d_{k}-\left[M_{k}+\mu_{k}\left(I+A^{T} D_{k}^{-1} A\right)\right]^{-1} r_{k}\right\| \\
& \left.\geq\left\|d_{k}\right\|-\| M_{k}+\mu_{k}\left(I+A^{T} D_{k}^{-1} A\right)\right]^{-1} r_{k} \| \\
& \geq\left\|d_{k}\right\|-\frac{\left\|r_{k}\right\|}{\mu_{k}} \\
& \geq\left\|d_{k}\right\|-\left\|m\left(x_{k}\right)^{T} \nabla m\left(x_{k}\right)\right\|\left\|F\left(x_{k}\right)\right\| \\
& \geq\left\|d_{k}\right\|-q \kappa_{g} L_{2}^{2}\left\|s_{k}\right\|^{2},
\end{aligned}
$$

By (5.19), we have that

$$
\left\|d_{k}\right\| \leq\left\|\tilde{d}_{k}\right\|+q \kappa_{g} L_{2}^{2}\left\|s_{k}\right\|^{2}
$$


Combining (5.16) and (5.20), we obtain that

$$
\left\|d_{k}\right\| \leq \hat{\kappa}\left\|s_{k}\right\|
$$

where $\hat{\kappa}=2 \theta_{2}$. The conclusion of lemma holds.

According to above Lemma, we know that there exists a positive constant $\hat{\kappa}$ such that (5.7) holds. The next lemma shows that for large enough $k$, the step size $\alpha_{k} \equiv 1, \lim _{k \rightarrow \infty} \theta_{k}=1$, and $=h_{k}=d_{k}$.

Lemma 5.3 Under Assumptions 4.1-4.4 and 5.1-5.5, the full stepsize $\alpha_{k}=1$ and $\theta_{k}=1$ is always accepted for $k$ sufficiently large so that $x_{k+1}=x_{k}+d_{k}$.

Proof First, we choose $\bar{k}$ sufficiently large such that Lemmas 5.1, 5.2, 5.3 and local error bound condition (5.4) hold for all $k \geq \bar{k}$. Because $X^{*} \subseteq L\left(x_{0}\right)$, we have that $A A^{T}+D\left(\bar{x}_{k}\right)$ is also a positive definite matrix, then there exists a constant $\kappa_{\hat{\Gamma}}>0$ such that $\left\|\hat{\Gamma}_{k}\right\| \leq \kappa_{\hat{\Gamma}}$. By Assumption 5.2, it follows from Step 2 of the algorithm that $Y_{k}$ is $\Lambda$-poised in $B\left(x_{k}, \beta\left|g_{\phi_{k}}^{T} \hat{\Gamma}_{k}\right|^{\frac{1}{2}}\right)$. Since $g_{\phi_{k}}=\nabla m\left(x_{k}\right)^{T} m\left(x_{k}\right)$, by (4.1), Lemma 4.2 and Lemma 4.3, we have that

$$
\begin{aligned}
\left\|J\left(x_{k}\right)-\nabla F\left(x_{k}\right)\right\| & =\left\|\nabla m\left(x_{k}\right)-\nabla F\left(x_{k}\right)\right\| \\
& \leq \sum_{i=1}^{q}\left\|\nabla m_{i}\left(x_{k}\right)-\nabla f_{i}\left(x_{k}\right)\right\| \\
& \leq q \kappa_{e g}\left|g_{\phi_{k}}^{T} \hat{\Gamma}_{k}\right|^{\frac{1}{2}} \\
& \leq q \kappa_{e g}\left\|g_{\phi_{k}}\right\|^{\frac{1}{2}}\left\|\hat{\Gamma}_{k}\right\|^{\frac{1}{2}} \\
& \leq q \kappa_{e g}\left\|\nabla m\left(x_{k}\right)\right\|^{\frac{1}{2}}\left\|m\left(x_{k}\right)\right\|^{\frac{1}{2}}\left\|\hat{\Gamma}_{k}\right\|^{\frac{1}{2}} \\
& =q \kappa_{e g}\left\|\nabla m\left(x_{k}\right)\right\|^{\frac{1}{2}}\left\|F\left(x_{k}\right)\right\|^{\frac{1}{2}}\left\|\hat{\Gamma}_{k}\right\|^{\frac{1}{2}} \\
& \leq L_{5}\left\|x_{k}-\bar{x}_{k}\right\|^{\frac{1}{2}},
\end{aligned}
$$

where $L_{5}=q \kappa_{e g} \sqrt{\kappa_{\hat{\Gamma}} q \kappa_{g} L_{2}}$.

According to Lemma 4.2, we have that

$$
\left\|F\left(x_{k}+d_{k}\right)-\left(F\left(x_{k}\right)+\nabla F\left(x_{k}\right) d_{k}\right)\right\| \leq \frac{L_{4} \sqrt{q}}{2}\left\|d_{k}\right\|^{2} .
$$

According to the definition of $m(x)$, we have that

$$
m\left(\bar{x}_{k}\right)=m\left(x_{k}\right)+\nabla m\left(x_{k}\right)\left(\bar{x}_{k}-x_{k}\right)+\frac{1}{2}\left(\bar{x}_{k}-x_{k}\right)^{T} \nabla^{2} m\left(\xi_{k}\right)\left(\bar{x}_{k}-x_{k}\right),
$$

where $\xi_{k} \in\left(x_{k}, \bar{x}_{k}\right)$.

By Assumption 4.2 and (5.23), we can obtain that

$$
\begin{aligned}
\left\|m\left(x_{k}\right)+\nabla m\left(x_{k}\right)\left(\bar{x}_{k}-x_{k}\right)\right\| & \leq \frac{1}{2}\left\|\nabla^{2} m\left(\xi_{k}\right)\right\|\left\|\bar{x}_{k}-x_{k}\right\|^{2} \\
& \leq \frac{\sqrt{q} \kappa_{H}}{2}\left\|\bar{x}_{k}-x_{k}\right\|^{2} .
\end{aligned}
$$

Let $s_{k}=\bar{x}_{k}-x_{k}$, by (5.24), we have that

$$
\begin{aligned}
\tilde{\phi}\left(s_{k}\right) & =\frac{1}{2}\left\|m\left(x_{k}\right)+\nabla m\left(x_{k}\right) s_{k}\right\|^{2}+\frac{1}{2} s_{k}^{T} A D_{k}^{-1}\left(C_{k}+\mu_{k} I\right) A s_{k}+\frac{1}{2} \mu_{k}\left\|s_{k}\right\|^{2} \\
& \leq \frac{\left(\sqrt{q} \kappa_{m}\right)^{2}}{8}\left\|s_{k}\right\|^{4}+\frac{\theta^{2} L_{2}^{2}\left\|C_{k}\right\|}{2}\left\|s_{k}\right\|^{2}+\frac{\left(1+\theta L_{2}\right) \mu_{k}}{2}\left\|s_{k}\right\|^{2} \\
& \leq \frac{\left(\sqrt{q} \kappa_{m}\right)^{2}}{8}\left\|s_{k}\right\|^{4}+\frac{q \theta^{2} \theta_{1} L_{2}^{3} \kappa_{g}}{2}\left\|s_{k}\right\|^{3}+\frac{\left(1+\theta L_{2}\right) \mu_{k}}{2}\left\|s_{k}\right\|^{2} \\
& \leq \frac{\left(\sqrt{q} \kappa_{m}\right)^{2}}{8}\left\|s_{k}\right\|^{4}+\frac{q \theta^{2} \theta_{1} L_{2}^{3} \kappa_{g}}{2}\left\|s_{k}\right\|^{3}+\frac{\left(1+\theta L_{2}\right)}{2} \zeta\left\|\hat{\Gamma}_{k}\right\|^{\frac{\delta}{2}}\left\|g_{\phi_{k}}\right\|^{\frac{\delta}{2}}\left\|s_{k}\right\|^{2} \\
& \leq 2 \kappa_{1}\left\|s_{k}\right\|^{3}+\kappa_{2}\left\|s_{k}\right\|^{2+\frac{\delta}{2}},
\end{aligned}
$$


where $\kappa_{1}=\frac{q \theta^{2} \theta_{1} L_{2}^{3} \kappa_{g}}{2}$ and $\kappa_{2}=\frac{\left(1+\theta L_{2}\right) \zeta\left(q \kappa_{\hat{\Gamma}} \kappa_{g} L_{2}\right)^{\frac{\delta}{2}}}{2}$.

Since $\tilde{d}_{k}$ is the solution of problem $\left(\hat{\hat{S}}_{k}\right)$, we have that

$$
\begin{aligned}
\left\|m\left(x_{k}\right)+\nabla m\left(x_{k}\right) \tilde{d}_{k}\right\|^{2} & \leq \tilde{\phi}_{k}\left(\tilde{d}_{k}\right) \leq \tilde{\phi}_{k}\left(s_{k}\right) \\
& \leq 2 \kappa_{1}\left\|s_{k}\right\|^{3}+\kappa_{2}\left\|s_{k}\right\|^{2+\frac{\delta}{2}} \\
& \leq 4\left(\kappa_{1}+\kappa_{2}\right)\left\|s_{k}\right\|^{2+\frac{\delta}{2}} .
\end{aligned}
$$

By (5.26), we have that

$$
\left\|m\left(x_{k}\right)+\nabla m\left(x_{k}\right) \tilde{d}_{k}\right\| \leq \kappa_{3}\left\|s_{k}\right\|^{1+\frac{\delta}{4}}
$$

where $\kappa_{3}=2 \sqrt{\kappa_{1}+\kappa_{2}}$.

Combining (3.7), (5.20) and (5.27), we have that

$$
\begin{aligned}
\left\|m\left(x_{k}\right)+\nabla m\left(x_{k}\right) d_{k}\right\| & \leq\left\|m\left(x_{k}\right)+\nabla m\left(x_{k}\right) \tilde{d}_{k}\right\|+\left\|\nabla m\left(x_{k}\right)\right\| \frac{\left\|r_{k}\right\|}{\mu_{k}} \\
& \leq \kappa_{3}\left\|s_{k}\right\|^{1+\frac{\delta}{4}}++q \kappa_{g} \frac{\left\|r_{k}\right\|}{\mu_{k}} \\
& \leq \kappa_{3}\left\|s_{k}\right\|^{1+\frac{\delta}{4}}+q^{2} \kappa_{g}^{2} L_{2}^{2}\left\|s_{k}\right\|^{2} \\
& \leq \kappa_{4}\left\|s_{k}\right\|^{1+\frac{\delta}{4}}
\end{aligned}
$$

where $\kappa_{4}=2 \kappa_{3}$.

According to Lemma 4.1, (5.7) and (5.28), we have that

$$
\begin{aligned}
\left\|F\left(x_{k}\right)+\nabla F\left(x_{k}\right) d_{k}\right\| & \leq\left\|m\left(x_{k}\right)+\nabla m\left(x_{k}\right) d_{k}\right\|+\left\|\nabla F\left(x_{k}\right)-\nabla m\left(x_{k}\right)\right\|\left\|d_{k}\right\| \\
& \leq \kappa_{4}\left\|s_{k}\right\|^{1+\frac{\delta}{4}}+L_{5} \hat{\kappa}\left\|s_{k}\right\|^{\frac{3}{2}} \\
& \leq 2 \kappa_{4}\left\|s_{k}\right\|^{1+\frac{\delta}{4}} .
\end{aligned}
$$

From (5.22) and (5.29), we have that

$$
\begin{aligned}
\left\|F\left(x_{k+1}\right)\right\| & \leq\left\|F\left(x_{k}\right)+\nabla F\left(x_{k}\right) d_{k}\right\|+\frac{\sqrt{q}}{2} L_{4}\left\|d_{k}\right\|^{2} \\
& \leq 2 \kappa_{4}\left\|s_{k}\right\|^{1+\frac{\delta}{4}}+\frac{\sqrt{q}}{2} L_{4} \hat{\kappa}^{2}\left\|s_{k}\right\|^{2} \\
& \leq \frac{2 \kappa_{4}}{\rho}\left\|F\left(x_{k}\right)\right\|^{1+\frac{\delta}{4}} .
\end{aligned}
$$

Because $\left\|s_{k}\right\| \rightarrow 0$ as $k \rightarrow+\infty$, there exists index $K$, when $k>K$, we have $\left\|s_{k}\right\|^{\frac{\delta}{2}} \leq \frac{(1-\gamma) \rho^{2}}{2 \kappa_{4}^{2}}$, by (5.30) and $\Phi\left(x_{k}\right)=$ $\frac{1}{2}\left\|F\left(x_{k}\right)\right\|^{2}$, for sufficiently large $k$, we obtain that

$$
\Phi\left(x_{k+1}\right) \leq(1-\gamma) \Phi\left(x_{k}\right) .
$$

Let $\hat{d}_{k}=D_{k}^{\frac{1}{2}} A d_{k}$, and $\left(d_{k} ; \hat{d}_{k}\right)$ is a solution of problem $\left(\hat{S}_{k}\right)$. Let the stepsize scalar $\alpha_{k}$ be given in (3.4) along $d_{k}$ to the boundary $\Omega$. By (3.4),

$$
\alpha_{k}=\min \left\{-\frac{a_{i} x_{k}-b_{i}}{a_{i}^{T} d_{k}} \mid-\frac{a_{i}^{T} x_{k}-b_{i}}{a_{i}^{T} d_{k}}>0, i=1,2, \cdots, l\right\},
$$


with $\alpha_{k}=+\infty$, if $-\frac{a_{i}^{T} d_{k}-b_{i}}{a_{i}^{T} d_{k}} \leq 0$ for all $i=1,2, \cdots, l$, then by (2.4), we have that

$$
a_{i}^{T} d_{k}=\left(a_{i}^{T} d_{k}-b_{i}\right)^{\frac{1}{2}} \hat{d}_{k}^{i}=-\frac{\left(a_{i}^{T} d_{k}-b_{i}\right) \hat{\lambda}_{k}^{i}}{\mu_{k}+\left|\hat{\lambda}_{k}^{i}\right|}
$$

where $\hat{d}_{k}^{i}$ and $\hat{\lambda}_{k}^{i}$ are the $i$ th component of the vectors $\hat{d}_{k}^{i}$ and $\hat{\lambda}_{k}$, respectively.

If $\mu_{k}=0$, since the strict complementarity of the problem $(P)$ holds at every limit point of $\left\{x_{k}\right\}$, i.e. $\left|\hat{\lambda}_{k}^{j}\right|+\mid a_{j}^{T} d_{k}-$ $b_{j} \mid>0$, for all large $k, \hat{\lambda}_{k}=\hat{\lambda}_{k}^{N}>0$ when $\hat{\lambda}_{k}=0$. So, $\hat{\lambda}_{k}^{i}=\left(\hat{\lambda}_{k}^{N}\right)^{i}>0$. From (5.32), it is clear that $\lim _{k \rightarrow \infty} \alpha_{k}=1$.

Otherwise, if $\mu_{k}>0$, by (5.32), we have that

$$
\alpha_{k}=-\frac{a_{j}^{T} x_{k}-b_{j}}{a_{j}^{T} d_{k}} \geq \frac{\mu_{k}+\left|\hat{\lambda}_{k}^{j}\right|}{\left|\hat{\lambda}_{k}^{j}\right|} \geq 1 .
$$

This means that the step size $\alpha_{k}=1$.

By (3.5) and (3.6), we have that $x_{k}+\alpha_{k} d_{k} \in \Omega$. Further, If $x_{k}+\alpha_{k} d_{k} \in \operatorname{int}(\Omega)$, then by Step 5 of the algorithm, we have that $\theta_{k}=1$,it follows from (3.2) and (5.31) that $\alpha_{k}=1$ holds when $k$ is sufficiently large. Then $h_{k}=d_{k}$ when $k$ is sufficiently large, which means $x_{k+1}=x_{k}+d_{k}$. Otherwise, $x_{k}+\theta_{k} \alpha_{k} d_{k} \in \operatorname{int}(\Omega)$, by the condition on the strictly feasible stepsize $\theta_{k} \in\left(\theta_{0}, 1\right]$, from some $0<\theta_{0}<1$ and $\theta_{k}-1=O\left(\left\|d_{k}\right\|\right), \lim _{k \rightarrow \infty} \theta_{k}=1$, comes from $\lim _{k \rightarrow \infty}\left\|d_{k}\right\|=0$. Therefore, we have also obtained $\theta_{k} \rightarrow 1$ as $\left\|d_{k}\right\| \rightarrow 0$. Then $x_{k+1}=x_{k}+d_{k} \in \Omega$ as $\alpha_{k}=1$ and $\theta_{k} \rightarrow 1$.

Lemma 5.4 Under Assumptions 4.1-4.4 and 5.1-5.5, for large enough $k$, there exists a positive constant $\vartheta$ such that

$$
\operatorname{dist}\left(x_{k+1}, X^{*}\right) \leq \vartheta \operatorname{dist}\left(x_{k}, X^{*}\right)^{\sigma},
$$

where $\sigma=1+\frac{\delta}{4}$.

Proof Let $s_{k}=\bar{x}_{k}-x_{k}$, similar to prove (5.30), we have that

$$
\left\|F\left(x_{k+1}\right)\right\| \leq \frac{2 \kappa_{4}}{\rho}\left\|F\left(x_{k}\right)\right\|^{1+\frac{\delta}{4}} \leq \kappa_{5}\left\|s_{k}\right\|^{1+\frac{\delta}{4}},
$$

where $\kappa_{5}=\frac{2 \kappa_{4}}{\rho} L_{2}^{1+\frac{\delta}{4}}$.

By (4.9), we can obtain that

$$
\left|g_{\phi_{k+1}}^{T} \hat{\Gamma}_{k+1}\right|^{\frac{1}{2}} \leq \kappa_{6}\left\|F\left(x_{k+1}\right)\right\|
$$

where $\kappa_{6}=q \kappa_{g}\left(\frac{\kappa_{D}^{\frac{1}{2}}\|A\|+\|A\|^{2}}{\lambda_{\min }\left[A A^{T}+D\left(\bar{x}_{k+1}\right)\right]}+1\right)$.

Similar to the proof in Lemma 4.6 [25], there exists a constant $\chi>0$ such that

$$
\left|\nabla \Phi\left(x_{k}\right)^{T} \Gamma_{k}-g_{\phi_{k}}^{T} \hat{\Gamma}_{k}\right| \leq \chi\left\|x_{k}-\bar{x}_{k}\right\| .
$$

By (5.36), we have that $\left|\nabla \Phi\left(x_{k}\right)^{T} \Gamma_{k}-g_{\phi_{k}}^{T} \hat{\Gamma}_{k}\right| \rightarrow 0$ for all large enough $k$.

From (5.4), for large enough $k$, we have that

$$
\left|g_{\phi_{k+1}}^{T} \hat{\Gamma}_{k+1}\right|^{\frac{1}{2}} \geq \rho\left\|x_{k+1}-\bar{x}_{k+1}\right\|=\operatorname{dist}\left(x_{k+1}, X^{*}\right) .
$$


Let $s_{k+1}=\bar{x}_{k+1}-x_{k+1}$, by (5.34), (5.35) and (5.37), we have that

$$
\begin{aligned}
\operatorname{dist}\left(x_{k+1}, X^{*}\right) & =\left\|s_{k+1}\right\| \\
& \leq \frac{1}{\rho}\left|g_{\phi_{k+1}}^{T} \Gamma_{k+1}\right|^{\frac{1}{2}} \\
& \leq \frac{\kappa_{6}}{\rho}\left\|F\left(x_{k+1}\right)\right\| \\
& \leq \vartheta\left\|s_{k}\right\|^{1+\frac{\delta}{4}}, \\
& =\vartheta \operatorname{dist}\left(x_{k}, X^{*}\right)^{\sigma},
\end{aligned}
$$

where $\vartheta=\frac{\kappa_{6} \kappa_{5}}{\rho}$ and $\sigma=1+\frac{\delta}{4}$.

According to the definition of parameter $\sigma$ given in (5.38), we know that $\sigma>1$. By Lemma 5.5, we can obtain the following convergence result for the distance function.

Theorem 5.5 Under Assumptions 4.1-4.4 and 5.1-5.5, let $\left\{x_{k}\right\}$ be a sequence generated by the proposed Algorithm. If $\sigma<2$ given in (5.38) holds, then the sequence $\left\{\operatorname{dist}\left(x_{k}, X^{*}\right)\right\}$ superlinear converges to zeros, i.e., the iterates $x_{k}$ approach the solution set $X^{*}$ at superlinear rate of local convergence.

In view of Theorem 5.5, we know that the distance $\operatorname{dist}\left(x_{k}, X^{*}\right)$ from the iterates $x_{k}$ to the solution set $X^{*}$ converges to zero locally superlinearly. Similar to prove Theorem 4.7 in [32], we have that the sequence $\left\{x_{k}\right\}$ proposed by the algorithm is convergent.

The following lemma shows the relationship between $\operatorname{dist}\left(x_{k+1}, X^{*}\right)$ and $\left\|d_{k}\right\|$ which produced by Step 3 of the algorithm.

Lemma 5.6 Under Assumptions 4.1-4.4 and 5.1-5.5, assume $x^{*}$ is a limit point of sequence $\left\{x_{k}\right\}$ generated by the proposed algorithm, the error estimate

$$
\left\|x_{k+1}-x^{*}\right\| \leq \frac{\varpi}{1-\varpi}\left\|x_{k+1}-x_{k}\right\|
$$

where $\varpi<1$ is a positive constant.

Proof By $\left(\hat{S}_{k}\right)$, let

$$
\hat{N}_{k}=\left[\begin{array}{cc}
\nabla m\left(x_{k}\right)^{T} \nabla m\left(x_{k}\right)+\mu_{k} I & 0 \\
0 & C_{k}+\mu_{k} I
\end{array}\right],
$$

according to the definition of $C_{k}$, we have that $\hat{N}_{k}$ is a positive semi-definite matrix. Similar to prove Lemma 3.1 in [34], we have that

$$
-g_{\phi_{k}}^{T} d_{k} \geq \tilde{\phi}_{k}(0)-\tilde{\phi}\left(d_{k}\right) \geq \frac{1}{2} \frac{\left|g_{\phi_{k}}^{T} \hat{\Gamma}_{k}\right|}{\left\|\hat{N}_{k}\right\|} .
$$

According to the definition of $\hat{N}_{k}$, we know that there exists a constant $\kappa_{N}>0$ such that $\left\|\hat{N}_{k}\right\| \leq \kappa_{N}$, by (5.40), we have that

$$
-g_{\phi_{k}}^{T} d_{k} \geq \frac{\left|g_{\phi_{k}}^{T} \hat{\Gamma}_{k}\right|}{2 \kappa_{N}}
$$

By (5.41), $\left\|g_{\phi_{k}}\right\| \leq\left\|\nabla m\left(x_{k}\right)\right\|\left\|m\left(x_{k}\right)\right\| \leq q \kappa_{g} L_{2}\left\|x_{k}-\bar{x}_{k}\right\|$ and (5.4), we obtain that

$$
q \kappa_{g} L_{2}\left\|x_{k}-\bar{x}_{k}\right\|\left\|d_{k}\right\| \geq \frac{\left|g_{\phi_{k}}^{T} \hat{\Gamma}_{k}\right|}{2 \kappa_{N}} \geq \frac{\rho^{2}}{2 \kappa_{N}}\left\|x_{k}-\bar{x}_{k}\right\|^{2} .
$$

By (5.42), we have that

$$
\left\|x_{k}-\bar{x}_{k}\right\|\left(q \kappa_{g} L_{2}\left\|d_{k}\right\|-\frac{\rho^{2}}{2 \kappa_{N}}\left\|x_{k}-\bar{x}_{k}\right\|\right) \geq 0
$$


Now, we will show that $q \kappa_{g} L_{2}\left\|d_{k}\right\| \geq \frac{\rho^{2}}{2 \kappa_{N}}\left\|x_{k}-\bar{x}_{k}\right\|$ holds. The proof is by contradiction. Suppose $q \kappa_{g} L_{2}\left\|d_{k}\right\|<$ $\frac{\rho^{2}}{2 \kappa_{N}}\left\|x_{k}-\bar{x}_{k}\right\|$, we obtain from (5.43) that

$$
\left\|x_{k}-\bar{x}_{k}\right\|=0
$$

which implies

$$
\left\|d_{k}\right\|<0
$$

This is a contradiction, so we have that

$$
\begin{aligned}
\left\|d_{k}\right\| & \geq \frac{\rho^{2}}{2 q \kappa_{N} \kappa_{g} L_{2}}\left\|x_{k}-\bar{x}_{k}\right\| \\
& =\frac{\rho^{2}}{2 q \kappa_{N} \kappa_{g} L_{2}} \operatorname{dist}\left(x_{k}, X^{*}\right)
\end{aligned}
$$

for all large enough $k$.

Let $s_{k}=\bar{x}_{k}-x_{k}$, by (5.2), (5.7) and (5.38), we have that

$$
\begin{aligned}
\left\|d_{k+1}\right\| & \leq \hat{\kappa} \operatorname{dist}\left(x_{k+1}, X^{*}\right) \\
& =\hat{\kappa}\left\|\bar{x}_{k+1}-x_{k+1}\right\| \\
& \leq 2 \hat{\kappa} \vartheta\left\|s_{k}\right\|^{\sigma} .
\end{aligned}
$$

For $\sigma$ given in (5.38), we have that $\sigma=1+\frac{\delta}{4}>1$. Since $\left\|s_{k}\right\| \rightarrow 0$ as $k \rightarrow+\infty$, we know that there exists a positive constant $\varpi<1$ such that $\frac{4 q \hat{\kappa} \kappa_{N} \kappa_{g} L_{2} \vartheta}{\rho^{2}}\left\|s_{k}\right\|^{\sigma-1} \leq \varpi$ for large enough $k$. Combining (5.45) and (5.46), we have that

$$
\left\|d_{k+1}\right\| \leq \frac{4 q \hat{\kappa} \kappa_{N} \kappa_{g} L_{2} \vartheta}{\rho^{2}}\left\|s_{k}\right\|^{\sigma-1}\left\|d_{k}\right\| \leq \varpi\left\|d_{k}\right\|,
$$

which means

$$
\left\|x_{k+1}-x_{k}\right\| \leq \varpi\left\|x_{k}-x_{k-1}\right\|
$$

Finally, we have that

$$
\begin{aligned}
\left\|x_{k+t+1}-x_{k+1}\right\| & \leq \sum_{j=k}^{k+t}\left\|x_{j+1}-x_{j}\right\| \\
& \leq \sum_{j=0}^{t} \varpi^{j+1}\left\|x_{k+1}-x_{k}\right\| \\
& \leq \frac{\varpi}{1-\varpi}\left\|x_{k+1}-x_{k}\right\| .
\end{aligned}
$$

If we take $t \rightarrow+\infty$, we obtain (5.39).

Theorem 5.7 Under Assumptions 4.1-4.4 and 5.1-5.5, the sequence $\left\{x_{k}\right\}$ generated by the algorithm converges to a point $x^{*} \in X^{*}$ with superlinear rate of local convergence.

Proof By (5.39) and (5.46), for all $k$ sufficiently large, we immediately obtain that

$$
\begin{aligned}
\left\|x_{k+1}-x^{*}\right\| & \leq \frac{\varpi}{1-\varpi}\left\|d_{k+1}\right\| \\
& \leq \frac{2 \varpi \hat{\kappa} \vartheta}{1-\varpi}\left\|s_{k}\right\|^{\sigma} \\
& \leq \kappa_{7}\left\|x_{k}-x^{*}\right\|^{\sigma}
\end{aligned}
$$


where $\kappa_{7}=\frac{4 \hat{\kappa} \vartheta}{1-\tilde{\omega}}$.

According to Assumption 5.3, we have taht $\delta \in[1,2]$, for $\sigma$ given in (5.38), we have that $\sigma=1+\frac{\delta}{4}<2$, then from (5.48), we obtain the sequence $\left\{x_{k}\right\}$ converges to $x^{*}$ with superlinear rate of local convergence.

By (2.4), we have that

$$
\left(\nabla m\left(x_{k}\right)^{T} \nabla m\left(x_{k}\right)+\mu_{k} I\right) d_{k}=-g_{\phi_{k}}+A^{T} \hat{\lambda}_{k},
$$

where $\hat{\lambda}_{k}=A^{T}\left[A A^{T}+D_{k}\right]^{-1} g_{\phi_{k}}$.

By (5.49), we have that

$$
\left(\nabla m\left(x_{k}\right)^{T} \nabla m\left(x_{k}\right)+\mu_{k} I\right) d_{k}=-\left(I-A^{T}\left[A A^{T}+D_{k}\right]^{-1} A\right) g_{\phi_{k}} .
$$

Let

$$
\left.\nabla m\left(x_{k}\right)\left(I-A^{T}\left[A A^{T}+D_{k}\right]^{-1} A\right]\right)=S_{k}
$$

and

$$
\begin{aligned}
N_{k}= & \left(\nabla m\left(x_{k}\right)^{T} \nabla m\left(x_{k}\right) A^{T}\left[A A^{T}+D_{k}\right]^{-1} A+A^{T}\left[A A^{T}+D_{k}\right]^{-1} A \nabla m\left(x_{k}\right)^{T} \nabla m\left(x_{k}\right)\right) \\
& -A^{T}\left[A A^{T}+D_{k}\right]^{-1} A \nabla m\left(x_{k}\right)^{T} \nabla m\left(x_{k}\right) A^{T}\left[A A^{T}+D_{k}\right]^{-1} A,
\end{aligned}
$$

we obtain that

$$
\begin{aligned}
S_{k}^{T} S_{k}= & \left(\nabla m\left(x_{k}\right)\left[I-A^{T}\left[A A^{T}+D_{k}\right]^{-1} A\right]\right)^{T} \nabla m\left(x_{k}\right)\left(I-A^{T}\left[A A^{T}+D_{k}\right]^{-1} A\right) \\
= & \left(I-A^{T}\left[A A^{T}+D_{k}\right]^{-1} A\right) \nabla m\left(x_{k}\right)^{T} \nabla m\left(x_{k}\right)\left(I-A^{T}\left[A A^{T}+D_{k}\right]^{-1} A\right) \\
= & \nabla m\left(x_{k}\right)^{T} \nabla m\left(x_{k}\right)-\left(\nabla m\left(x_{k}\right)^{T} \nabla m\left(x_{k}\right) A^{T}\left[A A^{T}+D_{k}\right]^{-1} A+A^{T}\left[A A^{T}+D_{k}\right]^{-1} A \nabla m\left(x_{k}\right)^{T} \nabla m\left(x_{k}\right)\right) \\
& +A^{T}\left[A A^{T}+D_{k}\right]^{-1} A \nabla m\left(x_{k}\right)^{T} \nabla m\left(x_{k}\right) A^{T}\left[A A^{T}+D_{k}\right]^{-1} A \\
= & \nabla m\left(x_{k}\right)^{T} \nabla m\left(x_{k}\right)-N_{k} .
\end{aligned}
$$

By (5.50), (5.51) and $g_{\phi_{k}}=\nabla m\left(x_{k}\right)^{T} m\left(x_{k}\right)=\nabla m\left(x_{k}\right)^{T} F\left(x_{k}\right)$, we have that

$$
\left(S_{k}^{T} S_{k}+\mu_{k} I+N_{k}\right) d_{k}=S_{k}^{T} F\left(x_{k}\right) .
$$

According to the definition of $N_{k}$, we know that $N_{k}$ is a symmetric matrix, by (5.52), we have that

$$
\mu_{k} I+N_{k}=Q_{k}^{T}\left(\mu_{k} I+\Lambda_{k}\right) Q_{k},
$$

where $Q_{k}$ is a orthogonal matrix and $\Lambda_{k}$ is a diagonal matrixes which the diagonal elements are the eigenvalues of $N_{k}$.

Let $\hat{S}_{k}=S_{k} Q_{k}^{T}$ and $\bar{d}_{k}=Q_{k} d_{k}$, then by (5.52), we have that

$$
\left(\hat{S}_{k}^{T} \hat{S}_{k}+\mu_{k} I+\Lambda_{k}\right) \bar{d}_{k}=\hat{S}_{k}^{T} F\left(x_{k}\right) .
$$

The singular value decomposition (SVD) of $\hat{S}\left(x^{*}\right)$ is

$$
\begin{aligned}
\hat{S}\left(x^{*}\right) & =U^{*} \Sigma^{*} V^{* T} \\
& =U^{*}\left[\begin{array}{cccccc}
\rho_{1}^{*} & & & & & \\
& \ddots & & & & \\
& & \rho_{r}^{*} & & & \\
& & & 0 & & \\
& & & & \ddots & \\
& & & & & 0
\end{array}\right] V^{* T} \\
& =U_{1}^{*} \Sigma_{1}^{*} V_{1}^{* T},
\end{aligned}
$$


where $\sigma_{1}^{*} \geq \sigma_{2}^{*} \geq \cdots \geq \sigma_{r}^{*}>0$ and $\operatorname{rank}\left(\Sigma_{1}^{*}\right)=r$. Suppose that the SVD decomposition of $\hat{S}_{k}$ is as follows:

$$
\begin{aligned}
& \hat{S}_{k}=U_{k} \Sigma_{k} V_{k}^{T} \\
& =\left(U_{k, 1} U_{k, 2} U_{k, 3}\right)\left[\begin{array}{ccccccccc}
\rho_{1}^{k} & & & & & & & \\
& \ddots & & & & & & \\
& & \rho_{r}^{k} & & & & & \\
& & & \rho_{r+1}^{k} & & & & & \\
& & & & \ddots & & & & \\
& & & & & \rho_{r+q}^{k} & & & \\
& & & & & 0 & & \\
& & & & & & \ddots & \\
& & & & & & & 0
\end{array}\right]\left[\begin{array}{c}
V_{k, 1}^{T} \\
V_{k, 2}^{T} \\
V_{k, 3}^{T}
\end{array}\right] \\
& =U_{k, 1} \Sigma_{k, 1} V_{k, 1}^{T}+U_{k, 2} \Sigma_{k, 2} V_{k, 2}^{T},
\end{aligned}
$$

where $\Sigma_{k, 1}, \Sigma_{k, 2}>0, \operatorname{rank}\left(\Sigma_{k, 1}\right)=r$ and $\operatorname{rank}\left(\Sigma_{k, 2}\right)=q \geq 0$. Consequently, (5.55) can be written as

$$
\hat{S}_{k}=U_{k, 1} \Sigma_{k, 1} V_{k, 1}^{T}+U_{k, 2} \Sigma_{k, 2} V_{k, 2}^{T} \text {. }
$$

For all large enough $k$, we assume that there exists a positive constant $L_{6}$ such that the matrix $\hat{S}_{k}$ satisfy the following conditions:

$$
\|\hat{S}(y)-\hat{S}(x)\| \leq L_{6}\|y-x\|, \quad \forall x, y \in B\left(x^{*}, \varepsilon_{1}\right)=\left\{y \mid\left\|x^{*}-y\right\| \leq \varepsilon_{1}\right\},
$$

where $\varepsilon_{1} \in(0,1)$. Let $x^{*}$ be a limit point of sequence $\left\{x_{k}\right\}$ generated by the proposed algorithm, if $I-A^{T}\left[A A^{T}+\right.$ $\left.D\left(x^{*}\right)\right]^{-1} A$ is a nonsingular matrix, we have the following lemma.

Lemma 5.8 Under Assumptions 4.1-4.4, 5.1-5.2 and 5.4-5.5, let $x^{*}$ be a limit point of sequence $\left\{x_{k}\right\}$ generated by the proposed algorithm, if $I-A^{T}\left[A A^{T}+D\left(x^{*}\right)\right]^{-1} A$ is a nonsingular matrix and the matrix $\hat{S}_{k}$ satisfy (5.56), then for large enough $k$, there exist positive constant $L_{7}, L_{8}$ and $L_{9}$ such that
(a) $\left\|U_{k, 1} U_{k, 1}^{T} F\left(x_{k}\right)\right\| \leq L_{7}\left\|x_{k}-\bar{x}_{k}\right\| ;$
(b) $\left\|U_{k, 2} U_{k, 2}^{T} F\left(x_{k}\right)\right\| \leq L_{8}\left\|x_{k}-x^{*}\right\|^{2}$;
(c) $\left\|U_{k, 3} U_{k, 3}^{T} F\left(x_{k}\right)\right\| \leq L_{9}\left\|x_{k}-\bar{x}_{k}\right\|^{2}$.

Proof According to Lemma 4.2, we know that

$$
\left\|F\left(x_{k}\right)-F\left(\bar{x}_{k}\right)\right\| \leq L_{2}\left\|x_{k}-\bar{x}_{k}\right\|,
$$

which means result $(a)$ holds.

By the theory of matrix perturbation [24] and (5.56), we have that

$$
\left\|\operatorname{diag}\left(\Sigma_{k, 1}-\Sigma_{1}^{*}, \Sigma_{k, 2}, 0\right)\right\| \leq\left\|\hat{S}_{k}-\hat{S}^{*}\right\| \leq L_{6}\left\|x_{k}-x^{*}\right\| .
$$

By (5.57), we have that

$$
\left\|\Sigma_{k, 1}-\Sigma_{1}^{*}\right\| \leq L_{6}\left\|x_{k}-x^{*}\right\|
$$

and

$$
\left\|\Sigma_{k, 2}\right\| \leq L_{6}\left\|x_{k}-x^{*}\right\| .
$$


By Lemma 4.2, we have that

$$
\begin{aligned}
\left\|F\left(x_{k}\right)+\nabla m\left(x_{k}\right)\left(\bar{x}_{k}-x_{k}\right)\right\| & =\left\|F\left(\bar{x}_{k}\right)-F\left(x_{k}\right)-\nabla m\left(x_{k}\right)\left(\bar{x}_{k}-x_{k}\right)\right\| \\
& \leq \frac{\left\|\nabla^{2} m\left(\xi_{k}\right)\right\|}{2}\left\|\bar{x}_{k}-x_{k}\right\|^{2} \\
& \leq \frac{q \kappa_{H}}{2}\left\|\bar{x}_{k}-x_{k}\right\|^{2},
\end{aligned}
$$

where $\xi_{k} \in\left(x_{k}, \bar{x}_{k}\right)$.

Let $p_{k}=-\hat{S}_{k}^{+} F\left(x_{k}\right)$, where $\hat{S}_{k}^{+}$is the pseudo-inverse of $\hat{S}_{k}$. It is the least squares solution of $\min \left\|F\left(x_{k}\right)+\hat{S}_{k} p\right\|$, so we obtain from (5.59) that

$$
\begin{aligned}
\left\|U_{k, 3} U_{k, 3}^{T} F\left(x_{k}\right)\right\| & =\left\|F\left(x_{k}\right)+\hat{S}_{k} p_{k}\right\| \\
& \leq\left\|F\left(x_{k}\right)+\nabla m\left(x_{k}\right)\left(I-A^{T}\left[A A^{T}+D_{k}\right]^{-1} A\right) Q_{k} Q_{k}^{T}\left(I-A^{T}\left[A A^{T}+D_{k}\right]^{-1} A\right)^{-1}\left(\bar{x}_{k}-x_{k}\right)\right\| \\
& =\left\|F\left(x_{k}\right)+\nabla m\left(x_{k}\right)\left(\bar{x}_{k}-x_{k}\right)\right\| \\
& \leq L 9\left\|\bar{x}_{k}-x_{k}\right\|^{2} .
\end{aligned}
$$

where $L_{9}=\frac{q \kappa_{H}}{2}$.

Let $\tilde{S}_{k}=\stackrel{U}{U}_{k, 1} \Sigma_{k, 1} V_{k, 1}^{T}$ and $\tilde{p}_{k}=-\tilde{S}_{k}^{+} F\left(x_{k}\right)$. Since $\tilde{p}_{k}$ is the least squares solution of $\min \left\|F\left(x_{k}\right)+\tilde{S}_{k} p\right\|$, it follows from (5.59) and (5.58) that

$$
\begin{aligned}
\left\|\left(U_{k, 2} U_{k, 2}^{T}+U_{k, 3} U_{k, 3}^{T}\right) F\left(x_{k}\right)\right\|= & \left\|F\left(x_{k}\right)+\tilde{S}_{k} \tilde{p}_{k}\right\| \\
\leq & \left\|F\left(x_{k}\right)+\tilde{S}_{k} Q_{k}^{T}\left(I-A^{T}\left[A A^{T}+D_{k}\right]^{-1} A\right)^{-1}\left(\bar{x}_{k}-x_{k}\right)\right\| \\
\leq & \left\|F\left(x_{k}\right)+\hat{S}_{k} Q_{k}^{T}\left(I-A^{T}\left[A A^{T}+D_{k}\right]^{-1} A\right)^{-1}\left(\bar{x}_{k}-x_{k}\right)\right\| \\
& +\left\|\left(\hat{S}_{k}-\tilde{S}_{k}\right) Q_{k}^{T}\left(I-A^{T}\left[A A^{T}+D_{k}\right]^{-1} A\right)^{-1}\left(\bar{x}_{k}-x_{k}\right)\right\| \\
\leq & L\left\|_{9}-x_{k}\right\|^{2}+\left\|U_{k, 2} \Sigma_{k, 2} V_{k, 2}^{T} Q_{k}^{T}\left(I-A^{T}\left[A A^{T}+D_{k}\right]^{-1} A\right)^{-1}\left(\bar{x}_{k}-x_{k}\right)\right\| \\
\leq & L{ }_{9}\left\|\bar{x}_{k}-x_{k}\right\|^{2}+L_{6}\left\|x_{k}-x^{*}\right\|\left\|\left(I-A^{T}\left[A A^{T}+D_{k}\right]^{-1} A\right)^{-1}\right\|\left\|\bar{x}_{k}-x_{k}\right\| \\
\leq & \tilde{\kappa}\left\|x_{k}-x^{*}\right\|^{2},
\end{aligned}
$$

where $\tilde{\kappa}=L_{9}+\hat{\lambda} L_{6}$ and $\hat{\lambda}=\sqrt{\lambda_{\min }\left(\left(I-A^{T}\left[A A^{T}+D\left(x^{*}\right)\right]^{-1} A\right)^{-T}\left(I-A^{T}\left[A A^{T}+D\left(x^{*}\right)\right]^{-1} A\right)^{-1}\right)}$.

Since $\left\|U_{k, 2} U_{k, 2}^{T} F\left(x_{k}\right)\right\|-\left\|U_{k, 3} U_{k, 3} F\left(x_{k}\right)\right\| \leq\left\|\left(U_{k, 2} U_{k, 2}^{T}+U_{k, 3} U_{k, 3}\right) F\left(x_{k}\right)\right\|$, we have from (5.60) that

$$
\begin{aligned}
\left\|U_{k, 2} U_{k, 2}^{T} F\left(x_{k}\right)\right\| & \leq\left\|U_{k, 3} U_{k, 3} F\left(x_{k}\right)\right\|+\tilde{\kappa}\left\|x_{k}-x^{*}\right\|^{2} \\
& \leq L_{8}\left\|x_{k}-x^{*}\right\|^{2}
\end{aligned}
$$

where $L_{8}=L_{9}+\tilde{\kappa}$.

Let $\tilde{s}_{k}=\left(I-A^{T}\left[A A^{T}+D_{k}\right]^{-1} A\right) d_{k}$, if there exist constant $\omega \in(0,1)$ and $\hat{\theta}>0$ such that $\left\|\Lambda_{k}\right\| \leq \omega \mu_{k}$ and $\| F\left(x_{k}+\right.$ $\left.d_{k}\right)\|\leq \hat{\theta}\| F\left(x_{k}+\tilde{s}_{k}\right) \|$, we can show that $\left\{x_{k}\right\}$ converges to $x^{*}$ quadratically.

Theorem 5.9 Under Assumptions 4.1-4.4, 5.1-5.2 and 5.4-5.5, assume that there exist constant $\omega \in(0,1)$ and $\hat{\theta}>0$ such that $\left\|\Lambda_{k}\right\| \leq \omega \mu_{k}$ and $\left\|F\left(x_{k}+d_{k}\right)\right\| \leq \hat{\theta}\left\|F\left(x_{k}+\tilde{s}_{k}\right)\right\|$ for all large enough $k$. Furthermore, assume that the condition of Lemma 5.8 holds, then the sequence $\left\{x_{k}\right\}$ converges to $x^{*}$ quadratically.

Proof Let $F_{k}=F\left(x_{k}\right)$, by (5.53), we have that

$$
\left(\hat{S}_{k}^{T} \hat{S}_{k}+\mu_{k} I+\Lambda_{k}\right) \bar{d}_{k}=\hat{S}_{k}^{T} F_{k}
$$

By the (SVD) of $\hat{S}_{k}$, we know that the step $\bar{d}_{k}$ is

$$
\bar{d}_{k}=-V_{k, 1}\left(\Sigma_{k, 1}^{2}+\mu_{k} I+\Lambda_{k, 1}\right)^{-1} \Sigma_{k, 1} U_{k, 1}^{T} F_{k}-V_{k, 2}\left(\Sigma_{k, 2}^{2}+\mu_{k} I+\Lambda_{k, 2}\right)^{-1} \Sigma_{k, 2} U_{k, 2}^{T} F_{k} .
$$


By (5.62), we have that

$$
\begin{aligned}
F_{k}+S_{k} d_{k}= & F_{k}+\hat{S}_{k} \bar{d}_{k} \\
= & F_{k}-U_{k, 1} \Sigma_{k, 1}\left(\Sigma_{k, 1}^{2}+\mu_{k} I+\Lambda_{k, 1}\right)^{-1} \Sigma_{k, 1} U_{k, 1}^{T} F_{k} \\
& \quad-U_{k, 2} \Sigma_{k, 2}\left(\Sigma_{k, 2}^{2}+\mu_{k} I+\Lambda_{k, 2}\right)^{-1} \Sigma_{k, 2} U_{k, 2}^{T} F_{k} \\
= & U_{k, 1}\left(\mu_{k} I+\Lambda_{k, 1}\right)\left(\Sigma_{k, 1}^{2}+\mu_{k} I+\Lambda_{k, 1}\right)^{-1} U_{k, 1}^{T} F_{k} \\
& +U_{k, 2}\left(\mu_{k} I+\Lambda_{k, 2}\right)\left(\Sigma_{k, 2}^{2}+\mu_{k} I+\Lambda_{k, 2}\right)^{-1} U_{k, 2}^{T} F_{k}+U_{k, 3} U_{k, 3}^{T} F_{k} .
\end{aligned}
$$

Since $\left\{x_{k}\right\}$ converges to $x^{*}$ superlinearly, we assume that $L_{6}\left\|x_{k}-x^{*}\right\|<\frac{\sigma_{r}^{*}}{2}$ holds for all large enough $k$. Then we obtain that

$$
\begin{aligned}
& \left\|\left(\Sigma_{k, 1}^{2}+\mu_{k} I+\Lambda_{k, 1}\right)^{-1}\right\| \\
\leq & \left\|\Sigma_{k, 1}^{-2}\right\| \leq \frac{1}{\left(\rho_{r}^{*}-L_{3}\left\|x_{k}-x^{*}\right\|\right)^{2}} \\
< & \frac{4}{\rho_{r}^{* 2}} .
\end{aligned}
$$

Because $\left\|\Lambda_{k}\right\| \leq \omega \mu_{k}$, we have that

$$
\left\|\left(\Sigma_{k, 2}^{2}+\mu_{k} I+\Lambda_{k, 2}\right)^{-1}\right\| \leq \frac{1}{(1-\omega) \mu_{k}} .
$$

The above two inequalities, together with (4.9), Lemma 4.2 and Lemma 5.9 imply that

$$
\begin{aligned}
\left\|F_{k}+\hat{S}_{k} \bar{d}_{k}\right\| & \leq \frac{8 L_{2}^{\delta} L_{6}}{\rho_{r}^{*}}\left\|x_{k}-x^{*}\right\|^{1+\delta}+\frac{L_{7}(1+\omega)}{1-\omega}\left\|x_{k}-x^{*}\right\|^{2}+L_{9}\left\|x_{k}-x^{*}\right\| \\
& \leq \kappa_{7}\left\|x_{k}-x^{*}\right\|^{2}
\end{aligned}
$$

where $\kappa_{7}=\frac{(1+\omega) L_{7}}{1-\omega}+\frac{8 L_{2}^{\delta} L_{6}}{\rho_{r}^{*}}+L_{9}$

According to the definition of $\tilde{s}_{k}$, we have that

$$
\begin{aligned}
\left\|F\left(x_{k}+\tilde{s}_{k}\right)\right\|= & \left.\| F_{k}+J_{k}\left(I-A^{T}\left[A A^{T}+D_{k}\right]^{-1}\right] A\right) d_{k} \\
& \left.\left.+\frac{1}{2} d_{k}^{T}\left(I-A^{T}\left[A A^{T}+D_{k}\right]^{-1}\right] A\right) \nabla m\left(x_{k}\right)^{T} \nabla^{2} m\left(\xi_{k}\right) \nabla m\left(x_{k}\right)\left(I-A^{T}\left[A A^{T}+D_{k}\right]^{-1}\right] A\right) d_{k} \| \\
\leq & \left\|F_{k}+\hat{S}_{k} \bar{d}_{k}\right\|+\kappa_{8}\left\|d_{k}\right\|^{2},
\end{aligned}
$$

where $\xi_{k} \in\left(x_{k}, x_{k}+\tilde{s}_{k}\right), \kappa_{8}=q \kappa_{H} \hat{\kappa}\left(1+\frac{q L_{3}\|A\|^{2}}{\lambda_{\min }\left[A A^{T}+D\left(x^{*}\right)\right]^{-1}}\right)$ and $\lambda_{\min }\left(\left[A A^{T}+D\left(x^{*}\right)\right]^{-1}\right)$ is the minimum eigenvalue of $\left[A A^{T}+D\left(x^{*}\right)\right]^{-1}$.

By (4.9), (5.4) and (5.65), we have that

$$
\begin{aligned}
\rho \operatorname{dist}\left(x_{k+1}, X^{*}\right) & \leq \kappa_{6}\left\|F\left(x_{k+1}\right)\right\| \\
& =\kappa_{6}\left\|F\left(x_{k}+d_{k}\right)\right\| \\
& \leq \kappa_{6} \hat{\theta}\left\|F\left(x_{k}+\tilde{s}_{k}\right)\right\| \\
& \leq \kappa_{6} \hat{\theta}\left\|F_{k}+\hat{S}_{k} \bar{d}_{k}\right\|+\kappa_{6} \kappa_{8} \hat{\theta}\left\|d_{k}\right\|^{2} \\
& \leq \kappa_{9}\left\|x_{k}-x^{*}\right\|^{2},
\end{aligned}
$$

where $\kappa_{9}=\kappa_{6} \hat{\theta}\left(\kappa_{7}+\kappa_{8} \hat{\kappa}\right)$.

From (5.45) and Lemma 5.2, we have that

$$
\left\|d_{k+1}\right\| \leq O\left(\left\|d_{k}\right\|^{2}\right),
$$


which implies that $\left\{x_{k}\right\}$ converges quadratically to $x^{*}$, namely,

$$
\left\|x_{k+1}-x^{*}\right\|=O\left(\left\|x_{k}-x^{*}\right\|^{2}\right) .
$$

The proof is completed.

\section{Numerical examples}

In this section, we want to illustrate the local behavior of the algorithm is section 3. The program code was written in MATLAB and run in MATLAB 7.0 environment. We terminated the iteration if

$$
\left|g_{\phi_{k}}^{T} \hat{\Gamma}_{k}\right|^{\frac{1}{2}} \leq 10^{-5} \text { or } k \geq k_{\max }
$$

Next, we will give some examples which the derivative information of the objective function is difficult to get. The numerical results of the 10 cases of variable dimension from 10 to 99 of Example 1 are given in Table 1. In Table 2 and Table 3, we give the numerical results of Example 2 and Example 3 which the 3 cases of variable dimension from 10 to 30, respectively. We test the Algorithm for "HS" problems which come from [16], our results are summarized in Table 4. In Table 4, $n$ denotes the dimension of the problem.

The results are summarized in Tables 1-4. In Tables 1-4, $n$ denotes the dimension of the problem. $q$ denotes the number of equations. The "— means that the corresponding algorithm can not reach the superlinear convergence.

Example 1

$$
\begin{aligned}
F(x) & =\left(f_{1}(x), \cdots, f_{q}(x)\right)=0 \\
\Omega & =\left\{\left(x_{1}, x_{2}, x_{3}\right) \mid 5-x_{3} \geq 0,100-x_{1} \geq 0,3 x_{1}-5 x_{2}+3 \geq 0,2 x_{1}+5 x_{2}+2 x_{3}-5 \geq 0\right\}
\end{aligned}
$$

where $f_{i}(x)=-0.01 i+\exp \left(-\frac{1}{x_{1}}\left(25+\left(-50 \ln (0.01 i)^{\frac{2}{3}}\right)-x_{2}\right)^{x_{3}}\right) \quad i=1,2, \cdots, q$.

Table 1: The computational results.

\begin{tabular}{cccccccc}
\hline$n$ & $q$ & $k$ & $\left\|x_{k}-x^{*}\right\|$ & $n$ & $q$ & $k$ & $\left\|x_{k}-x^{*}\right\|$ \\
\hline 3 & 10 & 1 & $3.2012 \mathrm{e}-02$ & 3 & 60 & 1 & $2.7856 \mathrm{e}-02$ \\
& & 2 & $2.1167 \mathrm{e}-03$ & & & 2 & $2.1149 \mathrm{e}-03$ \\
& & 3 & $3.2261 \mathrm{e}-04$ & & & 3 & $5.4351 \mathrm{e}-04$ \\
& & 4 & $7.3349 \mathrm{e}-06$ & & & 4 & $3.8961 \mathrm{e}-05$ \\
3 & 20 & 1 & $4.0562 \mathrm{e}-02$ & 3 & 70 & 1 & $3.1127 \mathrm{e}-03$ \\
& & 2 & $2.7134 \mathrm{e}-04$ & & & 2 & $2.2511 \mathrm{e}-05$ \\
& & 3 & $3.2679 \mathrm{e}-05$ & & & 3 & $3.2261 \mathrm{e}-06$ \\
& & 4 & $6.7731 \mathrm{e}-06$ & & & 4 & - \\
3 & 30 & 1 & $1.9546 \mathrm{e}-03$ & 3 & 80 & 1 & $2.8761 \mathrm{e}-02$ \\
& & 2 & $2.4562 \mathrm{e}-04$ & & & 2 & $3.5219 \mathrm{e}-03$ \\
& & 3 & $2.4421 \mathrm{e}-05$ & & & 3 & - \\
& & 4 & $3.3352 \mathrm{e}-06$ & & & 4 & - \\
3 & 40 & 1 & $8.4369 \mathrm{e}-03$ & 3 & 90 & 1 & $8.4635 \mathrm{e}-03$ \\
& & 2 & $2.5571 \mathrm{e}-04$ & & & 2 & $2.7791 \mathrm{e}-04$ \\
& & 3 & $3.2451 \mathrm{e}-06$ & & & 3 & $2.6731 \mathrm{e}-05$ \\
& & 4 & $3.2531 \mathrm{e}-07$ & & & 4 & $6.4572 \mathrm{e}-07$ \\
3 & 50 & 1 & $1.5862 \mathrm{e}-05$ & 3 & 99 & 1 & $2.7731 \mathrm{e}-03$ \\
& & 2 & $2.4536 \mathrm{e}-06$ & & & 2 & $4.9462 \mathrm{e}-04$ \\
& & 3 & $3.5521 \mathrm{e}-08$ & & & 3 & $5.5529 \mathrm{e}-05$ \\
& & 4 & - & & & 4 & $1.3023 \mathrm{e}-07$ \\
\hline
\end{tabular}




\section{Example 2}

$$
\begin{aligned}
F(x) & =\left(f_{1}(x), \cdots, f_{q}(x)\right)=0 \\
\Omega & =\left\{\left(x_{1}, x_{2}, x_{3}\right) \mid-2 x_{1}+3 x_{2}-1 \geq 0,50-x_{2} \geq 0,2 x_{1}+3 x_{2}-4 x_{3}-5 \geq 0\right\}
\end{aligned}
$$

where $f_{i}(x)=\exp \left(-x_{1} z_{i}\right)-x_{3} \exp \left(-x_{2} z_{i}\right)-y_{i}, z_{i}=0.1 i$ and $y_{i}=\exp \left(-z_{i}\right)-5 \exp \left(-10 z_{i}\right), i=1,2, \cdots, q$.

Table 2: The computational results.

\begin{tabular}{cccc}
\hline$n$ & $q$ & $k$ & $\left\|x_{k}-x^{*}\right\|$ \\
\hline 3 & 10 & 1 & $4.0327 \mathrm{e}-02$ \\
& & 2 & $2.4417 \mathrm{e}-03$ \\
& & 3 & $2.7938 \mathrm{e}-04$ \\
& & 4 & $2256 \mathrm{e}-06$ \\
3 & 20 & 1 & $1.9845 \mathrm{e}-03$ \\
& & 2 & $6.7329 \mathrm{e}-04$ \\
& & 3 & $3.7793 \mathrm{e}-05$ \\
& & 4 & $1.3352 \mathrm{e}-06$ \\
3 & 30 & 1 & $3.7681 \mathrm{e}-03$ \\
& & 2 & $2.7462 \mathrm{e}-04$ \\
& & 3 & $2.4531 \mathrm{e}-05$ \\
& & 4 & $4.8936 \mathrm{e}-06$ \\
\hline
\end{tabular}

\section{Example 3}

$$
\begin{aligned}
F(x) & =\left(f_{1}(x), \cdots, f_{q}(x)\right)=0 \\
\Omega & =\left\{\left(x_{1}, x_{2}, x_{3}\right) \mid-4 x_{1}+50 x_{2}-2 \geq 0,100-x_{2} \geq 0, x_{1}+20 x_{2}-3 x_{3}-1 \geq 0\right\}
\end{aligned}
$$

where $f_{i}=\exp \left(-i x_{1} / 10\right)-\exp \left(-x_{2} / 10\right)-x_{3}[\exp (-i / 10)-\exp (-i)], i=1,2, \cdots, q$.

Table 3: The computational results.

\begin{tabular}{cccc}
\hline$n$ & $q$ & $k$ & $\left\|x_{k}-x^{*}\right\|$ \\
\hline 3 & 10 & 1 & $6.2416 \mathrm{e}-03$ \\
& & 2 & $5.3214 \mathrm{e}-05$ \\
& & 3 & $8.9624 \mathrm{e}-06$ \\
& & 4 & $8.6782 \mathrm{e}-07$ \\
3 & 20 & 1 & $3.4462 \mathrm{e}-03$ \\
& & 2 & $3.2719 \mathrm{e}-04$ \\
& & 3 & $2.9763 \mathrm{e}-05$ \\
& & 4 & - \\
3 & 30 & 1 & $4.4438 \mathrm{e}-02$ \\
& & 2 & $6.1722 \mathrm{e}-03$ \\
& & 3 & $3.8746 \mathrm{e}-04$ \\
& & 4 & $5.5971 \mathrm{e}-05$ \\
\hline
\end{tabular}

Our test set consists of 14 zero residual problems. The "HS" problems in the test set are reformulated as leastsquares problems by simply taking the objective function as the first nonlinear function and taking the linear inequality constraints for the remaining nonlinear functions in the problem $(\mathrm{S})$.

Table 4 shows the local superlinear convergence of the test problems. In Table 1, we report the result of $\left\|x_{k}-x^{*}\right\|$ when the iterative point $x_{k}$ is close to the optimal solution $x^{*}$. From the result of the Table 4 , we can obtain that the algorithm has superlinear convergence in the most cases, but in some cases, the algorithm has quadratic convergence. For example, the test problem "HS229" shows that the algorithm has quadratic convergence from $k=2$ to $k=3$. 


\section{Final conclusions}

This paper has described the derivative-free affine scaling inexact Levenberg-Marquardt method for the solution of nonlinear equations subject to linear inequality constraints. The algorithm uses $2 n+1$ sampling points which is less than $\frac{(n+1)(n+2)}{2}$ required for building a fully quadratic model to asymptotically build at least fully linear models for each of the nonlinear functions in the problem. In the Levenberg-Marquardt method, it is assumed that the method is solved exactly at every iteration. However, it is expensive to take the exact solution of method for largescale problems. In this paper, we use inexact methods that find an approximate solution satisfying some appropriate conditions. Levenberg-Marquardt algorithms have to solve complicated quadratic programming subproblem at each iteration. In the case where $\Omega$ is polyhedral, it is difficulty in computation the search direction must satisfy strict interior feasibility. In order to overcome this shortcoming, we use line search technique in this paper. We have shown a local convergence rate of the algorithm under a suitable error bound condition, which is considerably weaker than a non-singularity assumption on Jacobian.

[1] S.Bellavia and B.Morini, Subspace trust-region methods for large bound-constrained nonlinear equations, SIAM J. Numer. Anal., 44 (2006) $1535-1555$.

[2] R.Behling and A.Fischer, A unified local convergence analysis of inexact constrained Levenberg-Marquardt methods. Optim. Lett., 6 (2012) 927C940.

[3] T.F.Coleman and Li, Y., A trust region and affine scaling interior point method for nonconvex minimization with linear inequality constraints, Math. Program., 19 (1997) 46-89.

[4] A.R.Conn, K.Scheinberg and L.N.Vicente, Geometry of interpolation sets in derivative free optimization, Math. Program., 111 (2008) 141 172.

[5] A.R.Conn, K.Scheinberg and PH.L.Toint, On the convergence of derivative-free methods for unconstrained optimization, in Approximation Theory and Optimization, Tributes to M.J.D.Powell, M.D.Buhmann and A.Iserles, eds., Cambridge University Press, Cambridge, UK, (1997) 83-108.

[6] H. Dan,N. Yamashita and M. Fukushima,Con vergence Properties of the Inexact Levenberg- Marquardt Method under Local Error Bound Conditions,T echnical Report 2001-001,Department of Applied Mathematics and Physics,Ky oto University (January 2001).

[7] I.I.Dikin, Iterative solution of problems of linear and quadratic programming, Soviet Math. Dokl. 8 (1967), pp.18-35.

[8] C.Elster and A.Neumaier, A grid algorithm for bound constrained optimization of noisy functions, IMA J. Numer. Anal., 15 (1995) $585-608$.

[9] M.E.Elhaway, Optimal Power Flow: Solution Techniques, Requirements and Challenges,IEEE Service Center,Piscataway,New Jersey,1996.

[10] A.Fischer, Local behavior of an iterative framework for generalized equations with nonisolated solutions, Math. Progr., 94 (2002) 91-124.

[11] A.Fischer, P.K.Shukla and M.Wang, On the inexactness level of robust Levenberg-Marquardt methods, Optimization, 59 (2010) $273-287$.

[12] C.A.Floudas and P.M.Pardalos(eds.), Encyclopedia of Optimization. 2nd edn. Springer, Berlin (2008)

[13] M.C.Ferris and J.S.Pang, Engineering and economic applications of complementarity problems, SIAM Rev. 39 (1997) 669-713.

[14] J.Fan and Y.Yuan, On the quadratic convergence of the Levenberg-Marquardt method without nonsingularity assumption, Computing., 74 (2005) 23-39.

[15] T.Glad and A.Goldstein, Optimization of functions whose values are subject to small errors, BIT, 17 (1977) 160-169.

[16] Hock, W., Schittkowski, K., Test examples for nonlinear programming codes. Lect. Notes Econ.Math.Syst. 187 (1981).

[17] C.Kanzow, N.Yamashita and M.Fukushima, Levenberg-Marquardt methods with strong local convergence properties for solving nonlinear equations with convex constraints, J. Comput. Appl. Math., 172 (2004) 375-397.

[18] K.Levenberg, A method for the solution of certain non-linear problems in least squares, Q. Appl. Math., 2 (1944) 164-168.

[19] M.Marazzi and J.Nocedal, Wedge trust region methods for derivative free optimization, Math. Program., 91 (2002) 289-305.

[20] M.Macconi, B.Morini and M.Porcelli, Trust-region quadratic methods for nonlinear systems of mixed equalities and inequalities, Appl. Numer. Math., 59 (2009) 859-876.

[21] D.W.Marquardt, An algorithm for least-squares estimation of nonlinear parameters, J. Soc. Ind. Appl. Math., 11 (1963) 431-441.

[22] Martinez, J.M., Qi, L.,Inexact Newton methods for solving nonsmooth equations, J. Comput. Appl. Math. 60 (1995) 127-145.

[23] M.J.D.Powell, Least Frobenius norm updating of quadratic models that satisfy interpolation conditions, Math. Program. Ser. B, 100 (2004) $183-215$.

[24] G.M.Stewart and J.G.Sun, Matrix perturbation theory, San Diego: Academic Press 1990.

[25] P.Wang and D.Zhu, An affine scaling derivative-free trust-region method for solving nonlinear systems subject to linear inequality constraints, International Journal of Computer Mathematics, DOI: 10.1080/00207160.2014.959942.

[26] T.Wang, R.D.C.Monteiro and K.J.S.Pang, An interior point potential reduction method for constrained equations, Math. Programming, 74 (1996) 159-195.

[27] A.J.Wood and B.F.Wollenberg, Power Generation, Operation and Control, John Wiley and Sons, New York, NY, 1996.

[28] N.Yamashita and M.Fukushima, On the rate of convergence of the Levenberg-Marquardt method, Computing (Suppl), 15 (2001) $227-238$.

[29] H.Zhang, A.R.Conn and K.Scheinberg, A derivative-free algorithm for least-squares minimization, SIAM, J. Optim, 20 (2010) 3555-3576.

[30] H.Zhang and A.R.Conn, On the local convergence of a derivative-free algorithm for least-squares minimization, Comput Optim, 51 (2012) 481-507.

[31] J.L.Zhang, On the convergence properties of the Levenberg-Marquardt method, Optimization, 52 (2003) 739-756.

[32] D.Zhu, Affine scaling interior Levenberg-Marquardt method for bound-constrained semismooth equations under local error bound conditions, J. Comput. Appl. Math., 219 (2008) 198-215.

[33] D.Zhu, An affine scaling trust-region algorithm with interior backtracking technique for solving bound-constrained nonlinear systems, Journal of Computational and Applied Matchematics, 184 (2005) 343-361.

[34] D.Zhu, Anew affine scaling interior point algorithm for nonlinear optimization subject to linear equality and inequality constraints, Journal of Computational and Applied Matchematics, 161 (2003) 1-25. 
Table 4: The computational results.

\begin{tabular}{|c|c|c|c|c|c|c|c|}
\hline Problem & $n$ & $\mathrm{k}$ & $\left\|x_{k}-x^{*}\right\|$ & Problem & $n$ & $\mathrm{k}$ & $\left|x_{k}-x^{*}\right|$ \\
\hline \multirow[t]{5}{*}{ HS38 } & 4 & 1 & $1.5000 \mathrm{e}-03$ & HS74 & 4 & 1 & $2.1000 \mathrm{e}-02$ \\
\hline & & 2 & $4.6745 e-04$ & & & 2 & $2.0713 \mathrm{e}-04$ \\
\hline & & 3 & $4.4168 \mathrm{e}-05$ & & & 3 & $4.1489 \mathrm{e}-05$ \\
\hline & & 4 & $3.8970 \mathrm{e}-07$ & & & 4 & $1.0365 \mathrm{e}-06$ \\
\hline & & 5 & $2.8019 \mathrm{e}-11$ & & & 5 & $4.1447 \mathrm{e}-08$ \\
\hline \multirow[t]{7}{*}{ HS53 } & 5 & 1 & $1.2000 \mathrm{e}-03$ & HS75 & 4 & 1 & $1.2000 \mathrm{e}-03$ \\
\hline & & 2 & $2.9141 \mathrm{e}-04$ & & & 2 & $9.0724 \mathrm{e}-04$ \\
\hline & & 3 & $5.8283 e-05$ & & & 3 & $3.6448 \mathrm{e}-05$ \\
\hline & & 4 & $5.8274 \mathrm{e}-06$ & & & 4 & $1.8224 \mathrm{e}-06$ \\
\hline & & 5 & $5.8275 \mathrm{e}-07$ & & & 5 & $9.1120 \mathrm{e}-07$ \\
\hline & & 6 & $5.8275 \mathrm{e}-08$ & & & 6 & $9.1123 \mathrm{e}-08$ \\
\hline & & 7 & $5.8284 \mathrm{e}-09$ & & & - & - \\
\hline \multirow[t]{5}{*}{ HS60 } & 3 & 1 & $1.3000 \mathrm{e}-03$ & HS81 & 5 & 1 & $2.0200 \mathrm{e}-02$ \\
\hline & & 2 & $7.2906 \mathrm{e}-04$ & & & 2 & $7.9335 \mathrm{e}-04$ \\
\hline & & 3 & $1.4578 \mathrm{e}-05$ & & & 3 & $3.9647 \mathrm{e}-07$ \\
\hline & & 4 & $7.8290 \mathrm{e}-06$ & & & 4 & $1.9823 \mathrm{e}-10$ \\
\hline & & 5 & $1.2576 \mathrm{e}-07$ & & & 5 & $8.6444 \mathrm{e}-11$ \\
\hline \multirow[t]{7}{*}{ HS63 } & 3 & 1 & $3.1000 \mathrm{e}-02$ & HS111 & 10 & 3 & $1.0665 \mathrm{e}-00$ \\
\hline & & 2 & $3.2735 \mathrm{e}-06$ & & & 2 & $8.5400 \mathrm{e}-02$ \\
\hline & & 3 & $6.5471 \mathrm{e}-07$ & & & 3 & $1.6000 \mathrm{e}-03$ \\
\hline & & 4 & $5.2376 \mathrm{e}-09$ & & & 4 & $8.1705 \mathrm{e}-04$ \\
\hline & & 5 & $8.3802 \mathrm{e}-12$ & & & 5 & $4.0847 \mathrm{e}-05$ \\
\hline & & 6 & $3.3521 \mathrm{e}-13$ & & & 6 & $1.6339 \mathrm{e}-07$ \\
\hline & & 7 & $6.7024 \mathrm{e}-14$ & & & 7 & $2.1740 \mathrm{e}-09$ \\
\hline \multirow[t]{7}{*}{ HS217 } & 2 & 1 & $4.9900 \mathrm{e}-02$ & HS231 & 2 & 2 & $4.9376 \mathrm{e}-03$ \\
\hline & & 2 & $4.9978 \mathrm{e}-04$ & & & 2 & $8.7656 \mathrm{e}-04$ \\
\hline & & 3 & $2.4989 \mathrm{e}-05$ & & & 3 & $3.5372 \mathrm{e}-05$ \\
\hline & & 4 & $1.2495 \mathrm{e}-08$ & & & 4 & $9.3562 \mathrm{e}-06$ \\
\hline & & 5 & $1.3259 \mathrm{e}-09$ & & & 5 & $6.4436 \mathrm{e}-09$ \\
\hline & & 6 & $3.4281 \mathrm{e}-11$ & & & - & - \\
\hline & & 7 & $4.4732 \mathrm{e}-12$ & & & - & - \\
\hline \multirow[t]{5}{*}{ HS224 } & 2 & 1 & $1.2643 \mathrm{e}-02$ & HS262 & 4 & 1 & $2.7000 \mathrm{e}-02$ \\
\hline & & 2 & $6.3461 \mathrm{e}-03$ & & & 2 & $4.7467 \mathrm{e}-03$ \\
\hline & & 3 & $7.8521 \mathrm{e}-05$ & & & 3 & $4.3321 \mathrm{e}-05$ \\
\hline & & 4 & $6.3361 \mathrm{e}-07$ & & & 4 & $6.4782 \mathrm{e}-06$ \\
\hline & & 5 & $2.5582 \mathrm{e}-08$ & & & 5 & $2.5531 \mathrm{e}-07$ \\
\hline \multirow[t]{7}{*}{ HS229 } & 2 & 1 & $1.5000 \mathrm{e}-02$ & HS265 & 4 & 2 & $6.7000 \mathrm{e}-02$ \\
\hline & & 2 & $2.3456 \mathrm{e}-04$ & & & 2 & $6.6454 \mathrm{e}-04$ \\
\hline & & 3 & $5.1042 \mathrm{e}-08$ & & & 3 & $3.2220 \mathrm{e}-05$ \\
\hline & & 4 & $5.8689 \mathrm{e}-09$ & & & 4 & $1.6111 \mathrm{e}-06$ \\
\hline & & 5 & $1.3259 \mathrm{e}-10$ & & & 5 & $2.0827 \mathrm{e}-09$ \\
\hline & & 6 & $2.4198 \mathrm{e}-11$ & & & 6 & $1.0463 \mathrm{e}-10$ \\
\hline & & 7 & $1.9902 \mathrm{e}-12$ & & & - & - \\
\hline
\end{tabular}

